\title{
Introduction to Cosmology and String Theory
}

Johanna Erdmenger and Martin Ammon

\section{1}

\section{Introduction}

Cosmology and string theory are two areas of fundamental physics which have progressed significantly over the last 25 years. Joining both areas together provides the possibility of finding microscopic explanations for the history of the early universe on the one hand, and of deriving observational tests for string theory on the other. In the subsequent seven chapters, different aspects of string cosmology are introduced and discussed.

This chapter contains a summary of the basics of both cosmology and string theory in view of providing a reference and glossary for the subsequent chapters. The basic concepts are introduced and briefly described, emphasizing those aspects which are used in the remainder of this book.

There is a wealth of excellent textbooks of both cosmology and string theory, to which readers interested in further details are referred to - for example [1-7]. Reviews on string cosmology include [8-11]. An introduction to string cosmology is found in the textbook [12].

Cosmology is introduced in Sections (1.2)-(1.4) below, and string theory in Sections (1.5)-(1.11).

\section{2}

\section{Foundations of Cosmology}

On the basis of experimental evidence, the common scenario of present-day cosmology is the model of the hot big bang, according to which the universe originated in a hot and dense initial state 13.7 billion years ago, and then has expanded and is still expanding. The most essential feature of the present-day universe is that it is homogeneous and isotropic, that is its structure is the same at every point and in every direction.

This "standard model of cosmology" has received substantial experimental backup, beginning with the discovery of the cosmic microwave background (CMB) ra- 
diation by Penzias and Wilson in 1964 [13]. In recent years, a wealth of precise data has been collected. We list just a few of the important new observations here: In the 1990s, observations of galaxies with the Hubble Space Telescope led in particular to an accurate measurement of the Hubble parameter. Fluctuations in the cosmic microwave background radiations have been observed with the COBE satellite, and subsequently with the BOOMERanG experiment. A further increase in precision came with the WMAP satellite launched in 2001, whose measurements of the parameters of the standard model of cosmology are consistent with the conclusion that the present-day universe is flat. Moreover, these measurements support the scenario of cosmic inflation. They will be supplemented by further data from the PLANCK satellite in the near future.

\subsection{1}

\section{Metric and Einstein Equations}

The homogeneity and isotropy of the universe is best described by the RobertsonWalker metric, which in $(-,+,+,+)$ signature commonly used in string theory reads

$$
\mathrm{d} s^{2}=-\mathrm{d} t^{2}+a^{2}(t)\left[\frac{\mathrm{d} r^{2}}{1-\kappa r}+r^{2}\left(\mathrm{~d} \theta^{2}+\sin ^{2} \theta \mathrm{d} \phi^{2}\right)\right] .
$$

Here $a(t)$ describes the relative size of space-like hypersurfaces at different times. $\kappa=+1,0,-1$ stands for positively curved, flat, and negatively curved hypersurfaces, respectively. The frequency of a photon traveling through the expanding universe experiences a redshift $z$ of the size

$$
1+z=\frac{\lambda_{\text {observed }}}{\lambda_{\text {emitted }}}=\frac{a_{\text {present }}}{a_{\text {emitted }}},
$$

where $\lambda$ denotes the photon wavelength

Using the scale factor $a(t)$ we define the Hubble parameter

$$
H \equiv \frac{\dot{a}}{a},
$$

with $\dot{a}(t)=\mathrm{d} a / \mathrm{d} t$. As was first discovered and suggested by Edwin Hubble, and has been verified with high precision by modern observational methods, the most distant galaxies recede from us with a velocity given by the Hubble law,

$$
v \simeq H d,
$$

where $d$ is the distance between us and the galaxies considered.

For describing the expanding universe it is often useful to use the term e-foldings, defined as $e \equiv \ln \left(a\left(t_{f}\right) / a\left(t_{\mathrm{i}}\right)\right)$, which describes the growth of the scale factor between some time $t_{\mathrm{i}}$ and a later time $t_{\mathrm{f}}$.

The dynamics governing the evolution of the scale factor $a(t)$ are obtained from inserting the Robertson-Walker metric into the Einstein equation

$$
R_{\mu \nu}-\frac{1}{2} R g_{\mu \nu}=8 \pi G T_{\mu \nu},
$$


where $R_{\mu v}$ and $R$ are the Ricci tensor and scalar, $G$ the Newton constant, and $T_{\mu \nu}$ the energy-momentum tensor. The universe is best described by the perfect fluid form for the energy-momentum tensor of cosmological matter, given by

$$
T_{\mu \nu}=(\varrho+p) u_{\mu} u_{v}+p g_{\mu \nu}
$$

where $u_{\mu}$ is the fluid four-velocity, $\varrho$ is the energy density in the rest frame of the fluid, and $p$ is the pressure in the same frame. For consistency with the RobertsonWalker metric, fluid elements are comoving in the cosmological rest frame, with normalized four-velocity

$$
u^{u}=(1,0,0,0) \text {. }
$$

The energy-momentum tensor is diagonal and takes the form

$$
T_{\mu \nu}=\left(\begin{array}{cc}
\varrho & \\
& p g_{i j}
\end{array}\right),
$$

where $g_{i j}$ stands for the spatial part of the Robertson-Walker metric, including the factor of $a^{2}(t)$. Inserting the Robertson-Walker metric (1.1) into the Einstein equation (1.5) with the energy-momentum tensor (1.6), we obtain the first Friedmann equation

$$
H^{2} \equiv\left(\frac{\dot{a}}{a}\right)^{2}=\frac{8 \pi G}{3} \sum_{j} \varrho_{j}-\frac{\kappa}{a^{2}},
$$

with the total energy density $\varrho=\sum_{j} \varrho_{j}$, where the sum is over all different types of energy density in the universe. Moreover, we have the evolution equation

$$
\frac{\ddot{a}}{a}+\frac{1}{2}\left(\frac{\dot{a}}{a}\right)^{2}=-4 \pi G \sum_{j} p_{j}-\frac{\kappa}{2 a^{2}} .
$$

Here $p_{j}$ labels the different types of momenta. Equations (1.9) and (1.10) may be combined into the second Friedmann equation

$$
\frac{\ddot{a}}{a}=-\frac{4 \pi G}{3} \sum_{j}\left(\varrho_{j}+3 p_{j}\right) .
$$

The first Friedmann equation may be used to define the critical energy density

$$
\varrho_{\mathrm{c}} \equiv\left(\sum_{j} \varrho_{j}\right)_{\mathrm{c}}=\frac{3 H^{2}}{8 \pi G} \simeq 10^{-29} \frac{\mathrm{g}}{\mathrm{cm}^{3}},
$$

for which $\kappa=0$ and space is flat. The density ratio

$$
\Omega_{\text {total }} \equiv \frac{\varrho}{\varrho_{\mathrm{c}}}
$$


thus allows us to relate the total energy density of the universe to its curvature behavior,

$$
\begin{aligned}
& \Omega_{\text {total }}>1 \Leftrightarrow \kappa=1, \\
& \Omega_{\text {total }}=1 \Leftrightarrow \kappa=0, \\
& \Omega_{\text {total }}<1 \Leftrightarrow \kappa=-1 .
\end{aligned}
$$

Recent WMAP observations have shown that today, $\Omega_{\text {total }}=1$ to great accuracy, which leads to the conclusion that the universe is flat.

Energy conservation, $\nabla^{\mu} T_{\mu \nu}=0$, gives the relation

$$
\dot{\varrho}+3 H(\varrho+p)=0 \text {. }
$$

This relation is not independent of the Friedmann equations. Using both of them, energy conservation (1.15) may be rewritten as

$$
\frac{\mathrm{d}}{\mathrm{d} t}\left(\varrho a^{3}\right)=-p \frac{\mathrm{d}}{\mathrm{d} t} a^{3} .
$$

\section{2 .2}

\section{Energy Content of the Universe}

There is good experimental evidence, in particular from WMAP measurements, that the cosmic fluid contains four different components, and that the total energy density $\varrho_{\text {total }}$ in the universe is equal to the critical density $\varrho_{\mathrm{c}}$ given by (1.12). This implies

$$
\Omega_{\text {total }}=\sum_{j} \Omega_{j}=1, \quad \Omega_{j}=\frac{\varrho_{j}}{\varrho_{c}},
$$

with $\Omega_{j}$ denoting the present-day fraction of the energy density contributed by the $j$-th fluid component. The four components of the cosmic fluid are the following: 1. Radiation: this component contains predominantly photons, most of which correspond to the cosmic microwave background. The photons are thermally distributed with temperature $T=2.715 \mathrm{~K}$. The gas of photons satisfies the equation of state

$$
p_{\text {Rad }}=\frac{1}{3} \varrho_{\text {Rad }} .
$$

Moreover, there are also cosmic relic neutrinos in this fluid component, thermally distributed with $T=1.9 \mathrm{~K}$. The total energy density of radiation is a small fraction,

$$
\Omega_{\mathrm{Rad}} \approx 8 \times 10^{-5},
$$

of the total present-day energy density.

2. Baryons: since their rest mass is much larger than their kinetic energy, their equation of state is

$$
p_{\mathrm{B}} \simeq 0 \text {. }
$$


Their energy fraction is

$$
\Omega_{\mathrm{B}} \approx 4 \% \text {. }
$$

3. Dark Matter: observations of galaxy movement and of matter influence on fluctuations in the CMB provide evidence that there has to be a large amount of long-lived nonrelativistic matter subject to gravitation, which is not detectable by its emitted radiation. Determining the exact structure of this dark matter remains one of the essential challenges of modern cosmology. Just as for the baryons, dark matter has the equation of state

$$
p_{\mathrm{DM}} \simeq 0,
$$

while its energy fraction is

$$
\Omega_{\mathrm{DM}} \approx 26 \%,
$$

so that the overall density of nonrelativistic matter is

$$
\Omega_{\mathrm{M}} \equiv \Omega_{\mathrm{B}}+\Omega_{\mathrm{DM}} \approx 30 \%
$$

4. Dark Energy: a fourth, similarly unexplained contribution to the cosmic fluid is dark energy, which for a total energy density $\Omega=1$, has to be present in the universe with the large fraction

$$
\Omega_{\mathrm{DE}} \approx 70 \%
$$

Its equation of state is expected to be

$$
p_{\mathrm{DE}}=-Q_{\mathrm{DE}} .
$$

Observational evidence that such a fluid component with negative pressure must be present include tests of the Hubble expansion rate using supernovae which imply that the overall expansion rate of the universe, the Hubble parameter $H=$ $\dot{a} / a$, is increasing at present. The Friedmann equation (1.11) implies that this can only happen for positive energy density if the total pressure is sufficiently negative, $p<-1 / 3 \varrho$. Since none of the other fluid components has negative pressure, a large fraction of such a component must be present.

Each of the above equations of state implies that $w_{j}=p_{j} / \varrho_{j}$ is time independent, with

$$
w_{\mathrm{Rad}}=\frac{1}{3}, \quad w_{\mathrm{M}}=0, \quad w_{\mathrm{DE}}=-1
$$

Inserting these values into the energy conservation condition in the form (1.16) we obtain, with $a_{0}$ the present-day value of $a$,

$$
\varrho_{j}=\varrho_{j, 0}\left(\frac{a_{0}}{a}\right)^{\alpha_{j}}, \quad \alpha_{j}=3\left(1+w_{j}\right)
$$


where

$$
\alpha_{\mathrm{Rad}}=4, \quad \alpha_{\mathrm{M}}=3, \quad \alpha_{\mathrm{DE}}=0 .
$$

The different equations of state for the different fluid components thus imply that their relative abundances differ in the past universe as compared to the present-day observations since their energy densities vary differently as the universe expands. The history of the universe splits into periods where radiation, matter, and dark energy dominate the evolution of the total density, consecutively. The transition between the radiation and matter-dominated regimes is called radiation-matter equality and occurs at a scale given by the comoving wave vector of magnitude $k \simeq(a H)_{\mathrm{eq}}$. Note also that the Friedmann equation (1.9) implies that for $w>-1 / 3$, the scale factor $a(t)$ grows more slowly than the Hubble scale $H^{-1}(t)$.

It is useful to define the comoving frame which moves along with the Hubble flow. A comoving observer is the only one which sees an isotropic universe.

\section{2 .3}

\section{Development of the Universe}

During its expansion the universe experienced a number of decisive physical events. The earliest cosmological event for which there is observational evidence is nucleosynthesis, which began about three minutes after the big bang, and lasted for about fifteen minutes. At this time, the universe cooled below $1 \mathrm{MeV}$ and light nuclei, hydrogen, helium, lithium, and beryllium, began to accumulate from protons and neutrons. The observational evidence for nucleosynthesis comes from measuring the relative abundance of these elements.

The radiation-matter crossover described above occurred at a redshift (1.2) of $z$ 3600 , or about 50000 years after the big bang. After this crossover, density inhomogeneities can grow only logarithmically with $a$ while they grow linearly with $a$ during radiation domination.

At a redshift of around $z \sim 1100$, or about 380000 years after the big bang, recombination of nuclei and electrons into electrically neutral atoms occurs. This is the origin of the cosmic microwave background which corresponds to the light which is free to move through the universe after recombination. Beforehand, photons interact with the charged medium surrounding them on short scales. The CMB corresponds to a surface of last scattering for the photons. Measurements of the CMB temperature fluctuations, which are of the order $\delta T / T \sim 10^{-5}$, provide direct information about the size of primordial density fluctuations at this time.

Finally, galaxy formation occurs in the universe once the primordial density fluctuations have been amplified to a scale at which they are no longer well-described by linear perturbations. According to the cold dark matter model (for reviews see for instance [14]), the distribution of galaxies observed today also requires the presence of nonrelativistic (cold) dark matter, together with nonlinear fluctuations. 


\section{3}

\section{Inflation}

\subsection{1}

\section{Puzzles Within the Big Bang Model}

When considering the initial conditions characterizing matter in the big bang scenario of an expanding universe, we encounter a number of puzzles. Three of them are discussed in the subsequent text. The initial conditions fix the matter distribution in the universe at the Planckian time of $t_{\mathrm{p}}=10^{-43} \mathrm{~s}$ when classical gravity becomes applicable.

Horizon problem. The horizon problem relates to the fact that the universe is so extremely homogeneous. The Friedmann equation (1.9) implies that the universe expands so quickly that thermal equilibration would violate causality. A dimensional analysis for the ratio $a_{\mathrm{i}} / a_{0}$ of the initial and the present-day value of the scale factor $a(t)$ shows that our universe was initially larger than a casual patch by a factor of the order $\dot{a}_{\mathrm{i}} / \dot{a}_{0}$. If expansion was always decelerated by an attractive gravity force, which implies $\dot{a}_{\mathrm{i}} / \dot{a}_{0} \gg 1$, then the homogeneity scale was always larger than the causality scale. In fact, using the present size of the universe, the Planck time and the temperature of the universe now and at Planck time, one finds that $\dot{a}_{\mathrm{i}} / \dot{a}_{0} \sim 10^{28}$. This would require an extraordinary fine tuning.

Flatness problem. While the horizon problem relates to the initial conditions for the spatial distribution of matter, the flatness problem relates to the initial velocities. These must satisfy the Hubble law (1.4). The ratio of the kinetic to the total energy of matter in the universe is again given by $\left(\dot{a}_{\mathrm{i}} / \dot{a}_{0}\right)^{2}$ and if this ratio is very large, a very unnatural fine tuning between the kinetic energy associated to Hubble expansion and the gravitational potential energy is required. This may be seen from the Friedmann equation (1.9) which implies

$$
\Omega(t)-1=\frac{\kappa}{(H a)^{2}},
$$

and thus, since the present-day $\Omega_{0}$ has been observed to be very close to unity,

$$
\Omega_{\mathrm{i}}-1=\left(\Omega_{0}-1\right)\left(\frac{\dot{a}_{0}}{\dot{a}_{\mathrm{i}}}\right)^{2}<10^{-56}
$$

for $\dot{a}_{\mathrm{i}} / \dot{a}_{0} \sim 10^{28}$. Such an astonishing fine-tuning appears implausible.

Initial perturbations. A third puzzle, related to the other two, concerns the origin of the original inhomogeneities needed to explain the large-scale structure of the present-day universe.

\subsection{2}

\section{The Concept of Inflation}

A concept which can solve the puzzles mentioned is inflation. The idea of inflation, first suggested in [15], is that there is an initial stage of accelerated expansion where 
gravity acts as a repulsive force. If gravity was always positive, then $\dot{a}_{\mathrm{i}} / \dot{a}_{0}$ is necessarily larger than one since gravity decelerates expansion. $\dot{a}_{\mathrm{i}} / \dot{a}_{0}<1$ is possible only if gravity is repulsive during some period of expansion. This period of repulsive gravity can in particular explain the creation of our universe from a single causally connected region. Moreover, since it accelerates expansion, small initial velocities inside a causally connected region become very large.

In the inflationary period we have $\ddot{a}>0$. From the Friedmann equation (1.11), which may be written in the form

$$
\ddot{a}=-\frac{4 \pi G}{3}(\varrho+3 p) a,
$$

for the total energy density $\varrho$ and the total momentum $p$. We read off that $\ddot{a}>0$ requires $\varrho+3 p<0$. This implies that the strong energy dominance condition, $\varrho+3 p>0$, must be violated during inflation. One example which violates this condition is a positive cosmological constant for which $p \approx-\varrho$.

Inflation can only appear during a limited period in time for consistency with cosmological observations. In simple inflationary models it takes place in the period of $t_{\text {inf }} \sim 10^{-36}-10^{-34} \mathrm{~s}$ after the big bang. It must end with a "graceful exit", after which $\ddot{a}$ becomes negative again.

Let us consider how the condition $p=-\varrho$ may be realized. The matter and its interactions during inflation is simply modeled by a single scalar field $\varphi=\varphi(t)$, the inflaton. This can be viewed as an order parameter describing the vacuum of the physical theory determining the very high energy physics. This gives rise to

$$
\varrho=\frac{1}{2} \dot{\varphi}^{2}+V(\varphi), \quad p=\frac{1}{2} \dot{\varphi}^{2}-V(\varphi),
$$

with potential $V(\varphi)$. The condition $p \approx-\varrho$ requires $\dot{\varphi}^{2} \ll V(\varphi)$, so the kinetic energy must be smaller than the potential energy. This is referred to as "slow roll". The Klein-Gordon equation or $\dot{\varrho}=-3 H(\varrho+p)$ imply

$$
\ddot{\varphi}+3 H \dot{\varphi}+V^{\prime}=0, \quad V^{\prime} \equiv \frac{\partial V}{\partial \varphi} .
$$

The second term in this equation corresponds to a friction term proportional to $H$. Generically if friction becomes large, we may neglect the second derivative term $\ddot{\varphi}$ and find an approximate asymptotic solution to (1.34). With $\ddot{\varphi}=0$, (1.34) implies

$$
\dot{\varphi} \approx-\left(\frac{V^{\prime}}{3 H}\right) .
$$

From the slow-roll condition $1 / 2 \dot{\varphi}^{2} \ll V$ we then obtain the two conditions

$$
\varepsilon \ll 1, \quad \eta \ll 1
$$

for the slow-roll parameters

$$
\varepsilon \equiv \frac{1}{2}\left(\frac{M_{\mathrm{p}} V^{\prime}}{V}\right)^{2}, \quad \eta \equiv \frac{M_{\mathrm{p}}^{2} V^{\prime \prime}}{V} .
$$


Single-field slow-roll inflation leads to important consequences for density fluctuations, as discussed in Section 1.4.3 below, and in further detail in Chapters 2 and 3. It turns out that these consequences can be described just in terms of the two small parameters $\varepsilon$ and $\eta$, together with the value of the Hubble parameter during inflation.

\section{4}

\section{Fluctuations}

\subsection{1}

\section{Characterization of Small Fluctuations}

An important question of cosmology is to study how the large-scale structure of the universe which is observed today, including galaxies and clusters of galaxies, developed from the initially flat and homogeneous universe. The large-scale structure has evolved from initially small fluctuations during the expansion of the universe. These small fluctuations are taken as initial conditions of the big bang model.

In linear approximation, the fluctuations of the Robertson-Walker metric (1.1), given by

$$
\mathrm{d} s^{2}=\left[{ }^{(0)} g_{\mu \nu}+\delta g_{\mu \nu}(x)\right] \mathrm{d} x^{\mu} \mathrm{d} x^{\nu},
$$

may be decomposed as follows using the symmetry properties of the unperturbed Robertson-Walker metric. The linear approximation applies to fluctuations on length scales below the Hubble scale. It implies that the different fluctuation modes decouple and have a Gaussian distribution.

The $\delta g_{00}$ component has the form

$$
\delta g_{00}=2 a^{2} \phi,
$$

with scalar $\phi$. The spacetime component $\delta g_{0 i}$ has the form

$$
\delta g_{0 i}=a^{2}\left(\partial_{i} B+S_{i}\right),
$$

where the index $i$ runs over the three space-like components. The vector $S_{i}$ satisfies $\partial_{i} S^{i}=0$. The fluctuation component $\delta g_{i j}$ is a tensor under 3-rotations and may be written as

$$
\delta g_{i j}=a^{2}\left(2 \psi \delta_{i j}+2 \partial_{i} \partial_{j} E+\partial_{i} F_{j}+\partial_{j} F_{i}+h_{i j}\right),
$$

where $\psi, E$ are scalars, $\partial_{i} F^{i}=0, h_{i}^{i}=0, \partial_{i} h_{j}^{i}=0$.

The fluctuations are thus described by the scalar fluctuations $\phi, \psi, B, E$, the vector fluctuations $S_{i}, F_{i}$, and the tensor fluctuations $h_{i j}$. The latter described gravitational waves. All of these functions change under coordinate reparametrizations, but may be regrouped into coordinate invariant expressions. A particularly important coordinate invariant combination of scalar fluctuations is

$$
\Phi \equiv \phi-\frac{1}{a}\left[a\left(B-E^{\prime}\right)\right]^{\prime},
$$


where the prime denotes differentiation with respect to conformal time $\eta$, defined by $\mathrm{d} t=a(t) \mathrm{d} \eta$. The fluctuation $\Phi$ is the relativistic equivalent of the Newton potential. The Einstein equation (1.5) relates the metric fluctuations to the energymomentum tensor and its fluctuations.

\section{4 .2}

\section{Power Spectrum}

The power spectrum of fluctuations $P(k)$ is obtained by transforming to Fourier space. In the linear approximation, a nonrelativistic Fourier transformation is appropriate (see for instance [16]). In particular, for the scalar fluctuations $\Phi$ of (1.42) we have

$$
\Phi(\boldsymbol{r}, t)=\int \frac{\mathrm{d}^{3} k}{(2 \pi)^{3}} \Phi_{k}(t) \exp [\mathrm{i}(\boldsymbol{k} / a) \cdot \boldsymbol{r}]
$$

where homogeneity and isotropy of the background imply that $\Phi_{k}(t)$ depends only on $k=|k|$ and $t$. $k$ is the wave vector in the comoving frame which moves along with the Hubble flow. The physical wavelength is $\lambda=2 \pi a / k$. The power spectrum is obtained from the autocorrelation function $\xi_{\Phi}(\boldsymbol{r})$,

$$
\xi_{\Phi}(\boldsymbol{r}) \equiv\langle\Phi(r) \Phi(0)\rangle=\int \frac{\mathrm{d}^{3} k}{(2 \pi)^{3}} P_{\mathrm{S}}(k) \exp [\mathrm{i}(\boldsymbol{k} / a) \cdot \boldsymbol{r}] .
$$

If we assume that the average denoted by the brackets \langle\rangle is given by a Gaussian distribution, we have

$$
P_{\mathrm{S}}(k) \equiv|\Phi(k)|^{2}
$$

for the power spectrum. A dimensionless measure of the power spectrum is obtained by performing an angular integration within the Fourier transformation,

$$
\langle\Phi(r) \Phi(0)\rangle=\int_{0}^{\infty} \frac{\mathrm{d} k}{k} \Delta_{\Phi}^{2}(k) \frac{\sin (k r / a)}{k r / a},
$$

with

$$
\Delta_{\mathrm{S}}^{2}(k) \equiv \frac{1}{2 \pi^{2}} k^{3} P_{\mathrm{S}}(k)
$$

The spectral index $n_{\mathrm{s}}$ is defined by

$$
n_{\mathrm{s}}-1 \equiv \frac{\mathrm{d} \ln \Delta_{\mathrm{s}}^{2}}{\mathrm{~d} \ln k}
$$


A spectral index of $n_{\mathrm{s}}=1$ corresponds to a scale invariant spectrum, also called a Harrison-Zel'dovich spectrum. For tensor fluctuations or gravitational waves, denoted by $h_{i j}$ in (1.41), the spectral index is defined by

$$
n_{\mathrm{T}} \equiv \frac{\mathrm{d} \ln \Delta_{\mathrm{T}}^{2}}{\mathrm{~d} \ln k}
$$

These fluctuations have not yet been observed.

The structure of the spectrum is influenced by the expansion of the universe. For instance, for a spectrum which is scale invariant for modes $k<k_{\mathrm{eq}}$, with $k_{\mathrm{eq}} \equiv$ $(a H)_{\mathrm{eq}}$ the momentum scale at radiation-matter equality, we have a spectrum of the form $\Delta_{\Phi}^{2}(k) \propto 1 / k^{4}$ for modes $k>k_{\text {eq }}$. This behavior arises since modes with $k>k_{\text {eq }}$ re-enter the Hubble scale before radiation-matter equivalence, while modes with $k<k_{\text {eq }}$ do so afterwards (remember that $a H$ shrinks during both matter and radiation dominated periods).

\subsection{3}

\section{Fluctuations and Inflation}

Inflation has a significant impact on the fluctuation spectrum which originates from two facts. First, there are new contributions to the equations of motion for the metric fluctuations which originate from fluctuations of the scalar inflaton field. Second, while the scale $a H$ shrinks during matter and radiation dominance, it grows during inflation, such that length scales $L \sim a$ grow faster than $H^{-1}$. There is a horizon corresponding to the graceful exit at which inflation ends, and where the length scales grow slower than the Hubble scale again.

In inflationary models, the fluctuations $\chi$ of the inflaton $\phi$ are obtained from linearizing the equation of motion

$$
\square \phi-V^{\prime}(\phi)=0
$$

after replacing $\phi \rightarrow \phi+\chi$ and linearizing in $\chi$ as well as in the metric fluctuations. The time dependence of the background forces $\chi$ and the scalar metric fluctuation $\Phi$ to mix with each other. For instance, for $k \gg a H$ the solution for $\Phi$ of the coupled equations shows a damped oscillation. In the opposite regime $k \ll a H$, the coupled fluctuations equations read, in the slow-roll approximation,

$$
3 H \dot{\chi}+V^{\prime \prime}(\phi) \chi+2 V^{\prime}(\phi) \Phi=0, \quad 2 M_{\mathrm{P}}^{2} H \Phi=\dot{\phi} \chi
$$

The solutions are, after transforming to momentum space,

$$
\chi_{k}=C_{k} \frac{V^{\prime}(\phi)}{V(\phi)}, \quad \Phi_{k}=-\frac{C_{k}}{2}\left(\frac{V^{\prime}(\phi)}{V(\phi)}\right)^{2},
$$

with $C_{k}$ a constant of integration. This constant is set by the initial conditions at the horizon where the universe exits the inflation period. Since all classical fluctuations 
are damped away during the inflation period, the new perturbations are fueled by quantum fluctuations. The quantization for the inflaton perturbations reads

$$
\chi(x)=\int \frac{\mathrm{d}^{3} k}{(2 \pi)^{3}}\left[c_{k} u_{k}(t) \exp (\mathrm{i} \boldsymbol{k} \cdot \boldsymbol{r} / a)+c_{k}^{*} u_{k}^{*}(t) \exp (-\mathrm{i} \boldsymbol{k} \cdot \boldsymbol{r} / a)\right],
$$

where $c_{k}^{*}, c_{k}$ are the creation and annihilation operators and $u_{k}(t) \exp (\mathrm{i} k \cdot \boldsymbol{r} / a)$ is a basis of eigenmodes of the background field equation. Evaluating $\chi_{k}$ at the horizon exit $t_{\text {he }}$, where $k=a H$, determines the integration constant to be

$$
C_{k}=u_{k}\left(t_{\mathrm{he}}\right)\left(\frac{V}{V^{\prime}}\right)_{\mathrm{he}} .
$$

Using this, the result for the power spectrum for the scalar metric fluctuations eventually reads

$$
\Delta_{\Phi}^{2}(k)=\left(\frac{V}{24 \pi^{2} M_{\mathrm{P}}^{4} \varepsilon}\right)_{\text {he }},
$$

with $\varepsilon$ the slow-roll parameter defined in (1.37).

For the spectral index (1.48) we obtain, using that

$$
\frac{\mathrm{d}}{\mathrm{d} \ln k}=-M_{\mathrm{P}}^{2}\left(\frac{V^{\prime}}{V}\right) \frac{\mathrm{d}}{\mathrm{d} \varphi}
$$

in the slow-roll approximation,

$$
n_{\mathrm{s}}-1=-6 \varepsilon+2 \eta,
$$

with $\varepsilon, \eta$ as in (1.37), where the right hand side is evaluated at horizon exit. This implies that $n_{\mathrm{s}}<1$. Therefore, inflation predicts a red tilt in the scalar power spectrum, since $n_{\mathrm{s}}<1$ means that the amplitude for smaller momentum modes is larger than the amplitude for larger momentum modes. Similarly, for tensor modes inflation predicts that

$$
\Delta_{\mathrm{T}}^{2}(k)=\frac{2 V}{3 \pi^{2} M_{\mathrm{P}}^{4}},
$$

and

$$
n_{\mathrm{T}}=-2 \varepsilon
$$

for the tensor spectral index (1.49).

This concludes our brief introduction to cosmology.

\section{5}

\section{Bosonic String Theory}

We now turn to the essentials of string theory, emphasizing aspects relevant to the subsequent chapters. We begin by discussing bosonic string theory, after which 
we focus on superstring theories and dualities between these theories. Moreover, the unification of consistent superstring theories in ten dimensions into M-theory will be discussed. Also, there is a short introduction to $D$-branes and to compactification scenarios of string theory to four spacetime dimensions. Finally, short introductions to string thermodynamics and to the AdS/CFT correspondence are given.

Whereas in conventional quantum field theory the elementary particles are point-like objects, the fundamental objects in perturbative string theory are onedimensional strings. As a string evolves in time, it sweeps out a two-dimensional surface in spacetime, the world-sheet of the string, which is the string counterpart of the world-line for a point particle. To parametrize the world-sheet of the string, two parameters are needed: the world-sheet time coordinate $\tau=\sigma^{0}$, which parametrizes the world-line in the case of a point-like particle, and $\sigma=\sigma^{1}$ parametrizing the spatial extent of the string. The embedding of the world-sheet of the fundamental string into the (target) spacetime is given by the functions $X^{\mu}(\tau, \sigma)$, which are also referred to as the embedding functions or target spacetime string coordinates. Since the action of a point-like particle is given by the length of the world-line, the natural generalization to the action of a string propagating through flat spacetime is given by the area of the world-sheet,

$$
S=-\frac{1}{2 \pi \alpha^{\prime}} \int \mathrm{d}^{2} \sigma \sqrt{-\operatorname{det} \partial_{\alpha} X^{\mu} \partial_{\beta} X_{\mu}},
$$

where $\mathrm{d}^{2} \sigma=\mathrm{d} \sigma^{0} \mathrm{~d} \sigma^{1}=\mathrm{d} \tau \mathrm{d} \sigma$. This is the Nambu-Goto action of a fundamental string. The determinant is taken with respect to $\alpha, \beta=0,1$, where $\alpha$ and $\beta$ label the world-sheet coordinates. Moreover, we use the short-hand notation $\partial_{\alpha}=\partial / \partial \sigma^{\alpha}$. The only free parameter appearing in this action is $\alpha^{\prime}$, which is related to the length of the string, $\alpha^{\prime}=l_{\mathrm{s}}^{2}$. The dimensionful prefactor $T=1 /\left(2 \pi \alpha^{\prime}\right)$ can be interpreted as the string tension or the energy per length. To get rid of the square root in the action of the fundamental string in view of quantization, an auxiliary field $h_{\alpha \beta}\left(\sigma^{0}, \sigma^{1}\right)$ is introduced, which has to satisfy the constraints given below. This gives rise to the Polyakov action,

$$
S=-\frac{1}{4 \pi \alpha^{\prime}} \int \mathrm{d}^{2} \sigma \sqrt{-h} h^{\alpha \beta} \partial_{\alpha} X^{\mu} \partial_{\beta} X_{\mu}
$$

which is classically equivalent to (1.60) using the equations of motion of $h_{\alpha \beta}$. In (1.61), $h$ is the determinant of the matrix $h_{\alpha \beta}$ and $h^{\alpha \beta}$ is the inverse matrix of $h_{\alpha \beta}$, that is $h^{\alpha \beta} h_{\beta \gamma}=\delta_{\gamma}^{\alpha}$. The auxiliary field $h_{\alpha \beta}$ is called the world-sheet metric. The Polyakov action is invariant under the following symmetries:

- Poincaré transformations

These transformations are global symmetries of the world-sheet fields $X^{\mu}$ of the form

$$
\delta X^{\mu}=\Lambda^{\mu}{ }_{v} X^{v}+a^{\mu} \quad \text { and } \quad \delta h^{\alpha \beta}=0,
$$

where $\Lambda_{v}^{\mu}$ and $a^{\mu}$ are Lorentz transformations and spacetime translations, respectively. 


\section{- Reparametrizations}

The Polyakov action is invariant under reparametrizations since a change in the world-sheet parametrization of the form $\sigma^{\alpha} \rightarrow f^{\alpha}(\sigma)=\sigma^{\prime \alpha}$ with

$$
h_{\alpha \beta}(\sigma)=\frac{\partial f^{\gamma}}{\partial \sigma^{\alpha}} \frac{\partial f^{\delta}}{\partial \sigma^{\beta}} h_{\gamma \delta}\left(\sigma^{\prime}\right) \quad \text { and } \quad X^{\prime \mu}\left(\tau^{\prime}, \sigma^{\prime}\right)=X^{\mu}(\tau, \sigma)
$$

does not change the action.

- Weyl transformations

The action is also invariant under rescalings of the world-sheet metric $h_{\alpha \beta}$

$$
h_{\alpha \beta} \rightarrow \mathrm{e}^{\omega(\sigma, \tau)} h_{\alpha \beta} \quad \text { and } \quad \delta X^{\mu}=0 .
$$

Since this transformation is a local symmetry of the action, the energymomentum tensor of the field theory defined on the world-sheet is traceless, that is $T_{a}^{a}=0$. After quantization, Weyl Symmetry is potentially broken by a conformal anomaly. In string theory, this anomaly has to be absent, which is only the case if the spacetime dimension of the target space is $D=26$ for bosonic string theory. Moreover, there are restrictions on the form of the background fields allowed (see Section 1.5.4).

The local symmetries may be used to choose a gauge which brings the components of the world-sheet metric into a simple form. In particular, the equations of motion of the action can be simplified by choosing the gauge

$$
h_{\alpha \beta}=\eta_{\alpha \beta}=\left(\begin{array}{cc}
-1 & 0 \\
0 & 1
\end{array}\right) .
$$

In this and other conformal gauges, the equation of motion for $X^{\mu}(\tau, \sigma)$ is a relativistic wave equation,

$$
\left(\partial_{\tau}^{2}-\partial_{\sigma}^{2}\right) X^{\mu}=0
$$

supplemented by the Virasoro constraints

$$
\begin{aligned}
\partial_{\tau} X^{\mu} \partial_{\sigma} X_{\mu} & =0, \\
\partial_{\tau} X^{\mu} \partial_{\tau} X_{\mu}-\partial_{\sigma} X^{\mu} \partial_{\sigma} X_{\mu} & =0 .
\end{aligned}
$$

These constraints are derived from the equations of motion of the auxiliary field $h_{\alpha \beta}$ in the Polyakov action and have to be satisfied to ensure the equivalence of the two actions (1.60) and (1.61) at the classical level.

\subsection{1}

\section{Open and Closed Strings}

By applying variational methods, it is possible to derive not only the equations of motion but also the possible boundary conditions for the string. There are two different types of strings: open and closed strings. 
1.5.1.1

\section{Closed Strings}

Closed strings are topologically equivalent to a circle, that is they do not have endpoints. If we parametrize these strings by the parameter $\sigma \in[0,2 \pi[$, the boundary conditions read

$$
X^{\mu}(\tau, 0)=X^{\mu}(\tau, 2 \pi), \quad \partial_{\sigma} X^{\mu}(\tau, 0)=\partial_{\sigma} X^{\mu}(\tau, 2 \pi), \quad h^{\alpha \beta}(\tau, 0)=h^{\alpha \beta}(\tau, 2 \pi) .
$$

This means that the string coordinates $X^{\mu}$ are periodic, that is the endpoints are joined to form a closed loop. The mode expansion for the closed string is simply given by a pair of left and right-moving waves, which travel around the string in opposite directions,

$$
X^{\mu}(\tau, \sigma)=X_{\mathrm{R}}^{\mu}(\tau-\sigma)+X_{\mathrm{L}}^{\mu}(\tau+\sigma) .
$$

$X_{\mathrm{R}}\left(X_{\mathrm{L}}\right)$ are the right (left) moving parts, respectively. The mode decompositions of the left and right-moving parts are given by

$$
X_{\mathrm{R}}^{\mu}(\tau-\sigma)=\frac{1}{2} x_{0}^{\mu}+\alpha^{\prime} p_{\mathrm{R}}^{\mu}(\tau-\sigma)+\mathrm{i} \sqrt{\frac{\alpha^{\prime}}{2}} \sum_{n \neq 0} \frac{1}{n} \alpha_{n}^{\mu} \mathrm{e}^{-2 \mathrm{i} n(\tau-\sigma)}
$$

and

$$
X_{\mathrm{L}}^{\mu}(\tau+\sigma)=\frac{1}{2} x_{0}^{\mu}+\alpha^{\prime} p_{\mathrm{L}}^{\mu}(\tau+\sigma)+\mathrm{i} \sqrt{\frac{\alpha^{\prime}}{2}} \sum_{n \neq 0} \frac{1}{n} \tilde{\alpha}_{n}^{\mu} \mathrm{e}^{-2 \mathrm{i} n(\tau+\sigma)} .
$$

$x_{0}^{\mu}$ and $p^{\mu}$ are the center-of-mass position and momentum of the string, respectively. The periodicity condition requires that $p_{\mathrm{R}}^{\mu}=p_{\mathrm{L}}^{\mu}$, and reality of $X^{\mu}$ requires the conditions $\alpha_{-n}^{\mu}=\left(\alpha_{n}^{\mu}\right)^{\star}$ and $\tilde{\alpha}_{-n}^{\mu}=\left(\tilde{\alpha}_{n}^{\mu}\right)^{\star}$. Moreover, the center-of-mass momentum $p^{\mu}$ can be identified with the zero mode of the expansion by

$$
\alpha_{0}^{\mu}=\tilde{\alpha}_{0}^{\mu}=\sqrt{\frac{\alpha^{\prime}}{2}} p^{\mu} .
$$

\subsubsection{2}

\section{Open Strings}

For open strings, two different boundary conditions in each direction $\mu$ of the spacetime are possible, Neumann or Dirichlet boundary conditions. In the case of Neumann boundary conditions, the component of the momentum normal to the boundary of the world-sheet vanishes, that is

$$
\partial_{\sigma} X_{\mu}(\tau, 0)=\partial_{\sigma} X_{\mu}(\tau, \pi)=0 .
$$

Note that the open string is now parametrized by $\sigma \in[0, \pi]$. The boundary condition implies that there is no momentum flowing through the ends of the string. The mode decomposition of the embedding function $X^{\mu}(\tau, \sigma)$ is given by

$$
X^{\mu}(\tau, \sigma)=x_{0}^{\mu}+2 \alpha^{\prime} p^{\mu} \tau+\mathrm{i} \sqrt{2 \alpha^{\prime}} \sum_{n \neq 0} \frac{1}{n} \alpha_{n}^{\mu} \mathrm{e}^{-i n \tau} \cos (n \sigma) .
$$


Because of the Neumann boundary condition, the left and right-moving waves of an open string are reflected into each other. As in the case of the closed string, the center-of-mass momentum $p^{\mu}$ of the string can be identified with the zero mode $\alpha_{0}^{\mu}$ of the expansion,

$$
\alpha_{0}^{\mu}=\sqrt{2 \alpha^{\prime}} p^{\mu} .
$$

If we choose Dirichlet boundary conditions along the $\mu$ direction of spacetime, the endpoints of the string are fixed, that is

$$
X^{\mu}(\tau, 0)=X^{\mu}(\tau, \pi)=x_{0}^{\mu},
$$

where $x_{0}^{\mu}$ is a constant. The mode decomposition is then given by

$$
X^{\mu}(\tau, \sigma)=x_{0}^{\mu}+\sqrt{2 \alpha^{\prime}} \sum_{n \neq 0} \frac{1}{n} \alpha_{n}^{\mu} \mathrm{e}^{-\mathrm{i} n \tau} \sin (n \sigma) .
$$

The string coordinate $X^{\mu}$ is real if the usual property $\left(\alpha_{n}^{\mu}\right)^{\star}=\alpha_{-n}^{\mu}$ holds. Note that the zero mode $\alpha_{0}^{\mu}$ is not present in directions where Dirichlet boundary conditions are imposed, since the center-of-mass momentum of the string vanishes.

The modern interpretation of open-string boundary conditions is that they correspond to hyperplanes, so-called $D p$-branes, on which open strings can end. In $p$ spatial dimensions and in the time direction, Neumann boundary conditions are implemented, whereas in the remaining $26-(p+1)$ dimensions Dirichlet boundary conditions are used. We will have a closer look at $D$-branes in Section 1.7.2 when we discuss T-duality and in Section 1.8. For more details on $D$-branes see the textbook [7].

\section{5 .2}

\section{Quantization}

The theory can be quantized by using the standard commutation relations for the fields $X^{\mu}$ and the momentum $P^{\mu}$, which is conjugate to $X^{\mu}$. These commutation relations imply commutation relations for creation and annihilation operators, $\alpha_{n}^{\mu}$ and $\tilde{\alpha}_{n}^{\mu}$, acting on the ground state of the fundamental string. ${ }^{1)}$

The masses squared $M^{2}$ of the excited states are

$$
M^{2}=\frac{1}{\alpha^{\prime}}(N-1)
$$

for open strings and

$$
M^{2}=\frac{2}{\alpha^{\prime}}(N+\bar{N}-2)
$$

1) We consider only noninteracting strings. The creation and annihilation operators can be considered as exciting internal degrees of freedom of the string. 
for closed strings. $N$ and $\bar{N}$ are the mass levels and are given by

$$
N=\sum_{n=1}^{\infty} \alpha_{-n}^{\mu} \alpha_{n \mu}, \quad \bar{N}=\sum_{n=1}^{\infty} \tilde{\alpha}_{-n}^{\mu} \tilde{\alpha}_{n \mu} .
$$

The mass levels $N$ and $\bar{N}$ have integer eigenvalues, which are also called $N$ and $\bar{N}$, respectively.

Physical string states of the closed string have to obey the level-matching condition $N=\bar{N}$ for the mass levels. Because of this condition, $\alpha_{n}^{\mu}$ and $\tilde{\alpha}_{n}^{\mu}$ are not independent. The spectrum of the closed string at the first two mass levels consists of

$-N=\bar{N}=0$ : tachyon with mass $M^{2}=-4 / \alpha^{\prime}$.

- $N=\bar{N}=1$ : a rank-two massless tensor field, which can be decomposed into an antisymmetric part $B_{\mu \nu}$ (the Kalb-Ramond field), a symmetric traceless part $g_{\mu \nu}$ (the graviton), and the trace of the symmetric part $\phi$ (the dilaton).

Since every string theory involves closed strings, a rank-two symmetric tensor field, which will be identified with the graviton in Section 1.5.4, is necessarily incorporated in string theory. Moreover, we will see that the vacuum expectation value of $\phi$ is related to the string coupling constant. The tachyon in the closed-string spectrum is much more severe since it may indicate an instability of the theory. Such a tachyon will not appear in the spectrum of closed superstrings if we demand that supersymmetry is not explicitly broken in the embedding space.

The physical states of the open string ending on a $D p$-brane at the first two mass levels are

- $N=0$ : A tachyon with $M^{2}=-1 / \alpha^{\prime}$ appears in the spectrum. According to Sen's conjectures [17], this is related to the fact that in bosonic string theories, $D$-branes are unstable and will decay to radiation of closed strings.

- $N=1$ : A massless vector boson. This will give rise to a $U(1)$ gauge theory on the $D p$ brane. Furthermore, if $p<25$ massless scalars for each direction normal to the $D p$-brane are found in the open-string spectrum.

\subsection{3}

\section{String Perturbation Theory: Interactions and Scattering Amplitudes}

The Feynman path integral is a very natural method for describing interactions in string theory. In this approach amplitudes are given by summing over all worldsheets, weighted by the factor $\exp (i S / \hbar)$, which connect initial and final string configurations, as shown in Figure 1.1 for the closed string.

For closed oriented strings, to which we restrict ourselves in this section, the sum is taken over all oriented two-dimensional world-sheets without boundaries.

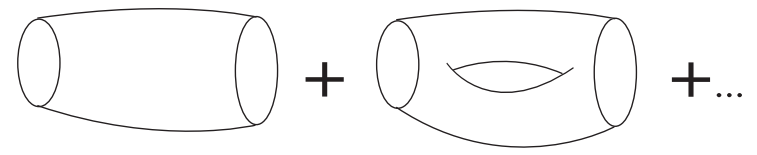

Figure 1.1 Examples of world-sheets connecting initial and final string configurations. 
To take open strings into account, world-sheets with boundaries have to be included. Interactions of the strings are already implicit in the sum over world-sheets. The world-sheet of a decay of one closed string into two is given in Figure 1.2. Thus, the world-sheet is similar to a Feynman diagram in which propagator lines are replaced by cylinders. A loop now corresponds to a handle of the world-sheet. An example is shown in Figure 1.3. The partition function $\mathcal{Z}$, that is the integral over all (Euclidean) world-sheet metrics $h_{\alpha \beta}$ and over all embeddings $X^{\mu}(\tau, \sigma)$ is given by, with $\hbar=1$,

$$
\mathcal{Z}=\int\left[\mathrm{d} X^{\mu}\right]\left[\mathrm{d} h_{\alpha \beta}\right] \exp (-S)
$$

The Euclidean action $S$ contains the usual Polyakov action $S_{\mathrm{p}}$, supplemented by a topological term weighting the different topologies of the string world-sheet $\Sigma$,

$$
S=S_{\mathrm{p}}+\lambda \chi \quad \text { with } \quad \chi=\frac{1}{4 \pi} \int_{\Sigma} \mathrm{d}^{2} \sigma \sqrt{h} R_{(h)},
$$

where $R_{(h)}$ is the Ricci scalar of the world-sheet metric $h_{\alpha \beta}$. Since $\chi$ is a topological term measuring the Euler number of the world-sheet, it does not contribute to the equations of motion. The factor $\exp (-\lambda \chi)$ in the path integral only affects the relative weighting of different topologies. Adding a handle to any world-sheet reduces the Euler number by two and therefore adds a factor of $\exp (2 \lambda)$. Since the process which is described by adding a handle corresponds to emitting and reabsorbing a closed string, the coupling constant of a closed string is given by $g_{\text {closed }}=\exp (\lambda)$. By analogous arguments, a string coupling constant $g_{\text {open }}$ of open strings can be introduced, which is related to $g_{\text {closed }}$ by

$$
\mathrm{g}_{\mathrm{s}} \equiv \mathrm{g}_{\text {closed }}=\mathrm{g}_{\text {open }}^{2}=\mathrm{e}^{\lambda} \text {. }
$$

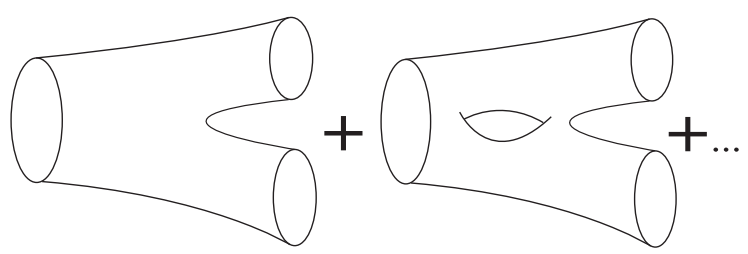

Figure 1.2 Joining and splitting of strings.
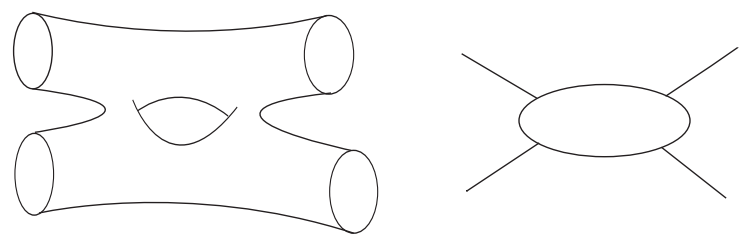

Figure 1.3 Comparison between Feynman diagrams of quantum field theory and interacting string diagrams. 


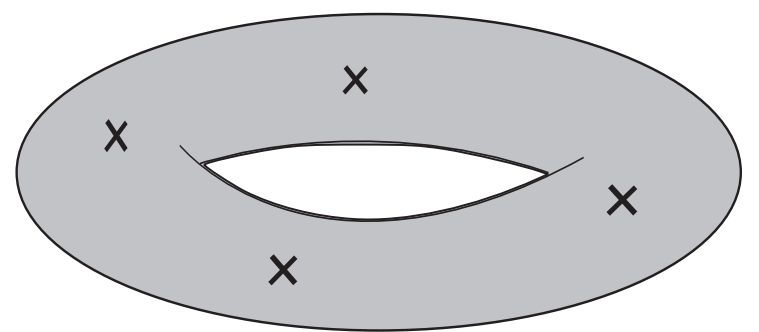

Figure 1.4 Four punctures on a torus. This diagram corresponds to Figure 1.3. The external string states (given by the four cylinders) in Figure 1.3 can be deformed to spikes. In this picture the spikes are removed and are represented by the punctures (crosses) on the torus.

In Section 1.5.4, we will see that the string coupling constant is fixed by the vacuum expectation value of the dilaton field.

Although it is a simple idea to sum over all world-sheets bounded by given initial and final curves, it is difficult to define this sum correctly and the resulting amplitudes are very complicated. To simplify scattering amplitudes, we take the limit where the string sources are at infinity. This is precisely a S-matrix element with specified incoming and outgoing strings which are on-shell and located at infinity. Under conformal transformations, the world-sheet can be transformed to a compact surface with $n$ points removed corresponding to the external string states. Such points are called punctures. By applying the path integral, the scattering amplitude is obtained by summing over all surfaces with $n$ punctures and by integrating at these punctures against the wave functions of the external string states. For detailed computational techniques see for instance [18] and standard textbooks on string theory [3-6].

\subsection{4}

\section{Bosonic String Theory in Background Fields}

Up to now we have considered the propagation of open and closed strings in Minkowski spacetime. By coupling the fundamental string to the massless closedstring excitations (see Section 1.5.2), strings propagating through curved spacetimes can be described. In particular we will see that the massless closed-string excitation $g_{\mu \nu}$ - which is traceless and symmetric - can be identified with the metric of the target spacetime. Since the quantized string theory in curved spacetime should be Weyl invariant, we obtain restrictions on the target spacetime allowed: the spacetime has to satisfy the vacuum Einstein equations (at least in the lowest order of $\alpha^{\prime}$ ). This is the goal of this section.

Now we will generalize the (Euclidean) Polyakov action in a simple manner to take into account couplings to the massless closed-string excitations: the antisym- 
metric tensor field $B_{(2)}$ (the components are called $\left.B_{\mu \nu}\right)^{2)}$, the symmetric traceless component $g_{\mu \nu}$ as well as the trace $\phi$. Since $g_{\mu \nu}$ is symmetric and traceless, the only possibility to couple it to the string (given by $X^{\mu}$ ) is

$$
S_{\mathrm{P}}=-\frac{1}{4 \pi \alpha^{\prime}} \int \mathrm{d}^{2} \sigma \sqrt{h} h^{\alpha \beta} \partial_{\alpha} X^{\mu} \partial_{\beta} X^{v} g_{\mu \nu}\left(X^{\varrho}\right) .
$$

We see that this equation is a generalization of the Polyakov action (1.61) to curved target spacetimes. Furthermore, we can couple the Kalb-Ramond field $B_{\mu \nu}\left(X^{\circ}\right)$ and the dilaton field $\phi\left(X^{\circ}\right)$ to the fundamental string by adding

$$
S_{\mathrm{B}, \phi}=\frac{1}{4 \pi \alpha^{\prime}} \int \mathrm{d}^{2} \sigma \sqrt{h}\left(\mathrm{i} \varepsilon^{\alpha \beta} \partial_{\alpha} X^{\mu} \partial_{\beta} X^{v} B_{\mu \nu}\left(X^{\varrho}\right)+\alpha^{\prime} R_{(h)} \phi\left(X^{\varrho}\right)\right)
$$

to the Polykov action (1.85), where $R_{(h)}$ is the Ricci scalar with respect to the worldsheet metric $h_{\alpha \beta}$. Comparing the dilaton dependent part of $S_{\mathrm{B}, \phi}$ to (1.83), we see that the dilaton sets the string coupling constant. Using (1.84) the string coupling constant $g_{\mathrm{s}}$ is given by

$$
g_{\mathrm{s}}=\mathrm{e}^{\phi} \text {. }
$$

Moreover, for ensuring Weyl invariance of the quantum theory (see remark after (1.64)), we impose the tracelessness of the energy-momentum tensor of the worldsheet theory in $D=26$ dimensions,

$$
T_{a}^{a}=-\frac{1}{2 \alpha^{\prime}} \beta_{\mu \nu}^{g} h^{a b} \partial_{a} X^{\mu} \partial_{b} X^{\nu}-\frac{\mathrm{i}}{2 \alpha^{\prime}} \beta_{\mu \nu}^{B} \varepsilon^{a b} \partial_{a} X^{\mu} \partial_{b} X^{\nu}-\frac{1}{2} \beta^{\phi} R,
$$

with

$$
\begin{aligned}
& \beta_{\mu \nu}^{g}=-\alpha^{\prime}\left(R_{\mu \nu}+2 \nabla_{\mu} \nabla_{\nu} \phi-\frac{1}{4} H_{\mu \varrho \sigma} H_{\nu}^{\varrho \sigma}\right)+\mathcal{O}\left(\alpha^{\prime 2}\right), \\
& \beta_{\mu \nu}^{B}=\alpha^{\prime}\left(-\frac{1}{2} \nabla^{\varrho} H_{\varrho \mu \nu}+\nabla^{\varrho} \phi H_{\varrho \mu \nu}\right)+\mathcal{O}\left(\alpha^{\prime 2}\right) \\
& \beta^{\phi}=\alpha^{\prime}\left(-\frac{1}{2} \nabla^{2} \phi+\nabla_{\varrho} \phi \nabla^{\varrho} \phi-\frac{1}{24} H_{\varrho \mu \nu} H^{\varrho \mu \nu}\right)+\mathcal{O}\left(\alpha^{\prime 2}\right)
\end{aligned}
$$

to the lowest order of $\alpha^{\prime} . H_{\varrho \mu \nu}$ are the components of the field strength $H_{(3)}$ of the Kalb-Ramond field $B_{(2)}$, that is

$$
H_{\varrho \mu \nu}=\partial_{\varrho} B_{\mu v}+\partial_{\mu} B_{v \varrho}+\partial_{\nu} B_{\varrho \mu} .
$$

The Polyakov action leads to a Weyl-invariant quantum theory if all three functions $\beta_{\mu \nu}^{g}, \beta_{\mu \nu}^{B}$ and $\beta^{\phi}$ vanish. Remarkably, the consistency equations $\beta_{\mu \nu}^{g}=\beta_{\mu \nu}^{B}=\beta^{\phi}=0$ can be derived as equations of motion from the target spacetime action

$$
S=\frac{1}{2 \kappa_{0}} \int \mathrm{d}^{26} X \sqrt{-g} \mathrm{e}^{-2 \phi}\left[R+4 \nabla_{\mu} \phi \nabla^{\mu} \phi-\frac{1}{12} H_{\mu \nu \varrho} H^{\mu \nu \varrho}+\mathcal{O}\left(\alpha^{\prime}\right)\right] .
$$

2) In this chapter we denote the number of indices of an antisymmetric tensor field by numbers in brackets. Therefore $B_{(2)}$ has two indices. If we exchange the indices, we obtain a minus sign, that is $B_{\mu \nu}=-B_{\nu \mu}$. Since the number of indices are apparent in the component notation, this number is omitted. 
This is the effective action for the massless string states $B_{\mu \nu}, g_{\mu v}$, and $\phi$ of the closed-string sector, where the effects due to the tachyon are omitted. Here $R$ is the Ricci scalar of the symmetric tensor field $g_{\mu \nu}$ and $\nabla_{\mu}$ are the covariant derivatives.

As discussed above, the string coupling constant is given by the expectation value of the dilaton $g_{s}=\mathrm{e}^{\phi}$. Moreover, the massless rank-two symmetric tensor field $g_{\mu \nu}$ can be identified with the graviton since $g_{\mu \nu}$ has to satisfy the equations of motion $\beta_{\mu \nu}^{g}=0$, which also follow immediately from the effective action. The first term in (1.93) is an Einstein-Hilbert term coupled to a dilaton. Therefore, $g_{\mu v}$ is identified with the target spacetime metric (see also (1.85)).

Moreover, we can canonically normalize the Einstein-Hilbert term of the action (1.93). Rescaling the metric ${ }^{3)}$

$$
\widetilde{\mathrm{g}}_{\mu \nu}=\mathrm{e}^{\frac{1}{6}\left(\phi_{0}-\phi\right)} \mathrm{g}_{\mu \nu},
$$

the action (1.93) can be rewritten in the form

$$
S=\frac{1}{2 \kappa^{2}} \int \mathrm{d}^{26} X \sqrt{-\tilde{\mathrm{g}}}\left[\tilde{R}-\frac{1}{6} \nabla_{\mu} \tilde{\phi} \nabla^{\mu} \tilde{\phi}-\frac{1}{12} \mathrm{e}^{-\frac{1}{3} \tilde{\phi}} H_{\mu \nu \varrho} H^{\mu \nu \varrho}+\mathcal{O}\left(\alpha^{\prime}\right)\right],
$$

with $\tilde{\phi}=\phi-\phi_{0}$ and $\kappa=\kappa_{0} \mathrm{e}^{\phi_{0}}=\sqrt{8 \pi G_{N}}$. Looking at the part involving the Ricci scalar $\tilde{R}$, which is determined by the rescaled metric $\tilde{g}_{\mu \nu}$, we see that we have removed the factor involving the dilaton $\phi$ in the Einstein-Hilbert part of the action (1.95). Whereas the action written in terms of the original fields is called the string-frame action, the latter, canonically normalized action is referred to as the Einstein-frame action.

In view of coupling the open string to the Abelian gauge field $A_{\mu}$ living on a $D$ brane, we have to include a term of the form

$$
S_{\mathrm{A}}=\int_{\partial \Sigma} \mathrm{d} \tau A_{\mu}(X) \partial_{\tau} X^{\mu},
$$

where $\partial \Sigma$ denotes the boundary of the world-sheet $\Sigma$. The effective action of the open-string sector, summarizing the leading order (in $\alpha^{\prime}$ ) open-string physics at tree level, is given by ${ }^{4}$

$$
S=-C \int \mathrm{d}^{26} X \mathrm{e}^{-\phi} \operatorname{Tr} F_{\mu \nu} F^{\mu \nu}
$$

where $C$ is a dimensionful constant. Therefore, the physics of the open-string sector at tree level is described by Yang-Mills theories. In the case of one $D$-brane the gauge group is $U(1)$, but can be generalized to non-Abelian gauge groups. In the Section 1.8 .1 we will discuss the effective action of $D$-branes, which determines the open-string physics.

3) The rescale of the metric depends on the dimension $D$ of the target spacetime. For simplicity we used here $D=26$.

4) For details on how to compute the effective $D$-brane action see [19]. 


\section{5 .5}

\section{Chan-Paton Factors}

So far we have seen that open strings on one $D p$-brane are described by a $U(1)$ gauge theory. In order to generalize this to non-Abelian gauge theories, ChanPaton factors are introduced on a stack of coincident $N$ Dp-branes. Chan-Paton factors are nondynamical degrees of freedom from the world-sheet point-of-view, which are assigned to the endpoints of the string. These factors label the open strings that connect the various coincident $D$-branes. For example, the Chan-Paton factor $\lambda_{i j}$ labels strings stretching from brane $i$ to brane $j$, with $i, j \in\{1, \ldots, N\}$. The resulting matrix $\lambda$ is an element of a Lie algebra. It turns out that the only Lie algebra consistent with open-string scattering amplitudes is $U(N)$ in the case of oriented strings, where $N$ is the number of coincident $D$-branes. Therefore, $\lambda$ can be chosen as a Hermitean matrix and $\lambda_{i j}$ are the corresponding entries of the matrix.

Although the Chan-Paton factors are global symmetries of the world-sheet action, the symmetry turns out to be local in the target spacetime. The theory of open strings ending on coincident $D$-branes can effectively be described by a nonAbelian gauge theory. For more details see [7].

\subsection{6}

\section{Oriented Versus Unoriented Strings}

So far we have considered oriented strings only. By oriented, we mean that a left to right direction on the string may be unambiguously defined. This is obvious since we parametrize the spatial extent by $\sigma$. Unoriented strings are constructed by imposing the world-sheet parity transformation $\Omega$,

$$
\Omega: \sigma \rightarrow \sigma_{0}-\sigma
$$

where $\sigma_{0}=2 \pi$ for closed and $\sigma_{0}=\pi$ for open strings. This transformation, which changes the orientation of the world-sheet, is a global symmetry of string theory. We can consistently truncate the theory by using only $\Omega$-invariant string states. The corresponding theories are called unoriented string theories.

Focusing on massless string states, the open-string vector boson has eigenvalue $\Omega=-1$, as well as the Kalb-Ramond field of the closed sector. Both are projected out of the spectrum of unoriented string theories. But if nontrivial Chan-Paton factors are introduced, the vector boson will be present after the $\Omega$-projection and give rise to a $S O(N)$ or $S p(N)$ gauge theory. Unfortunately, the tachyons of the open and closed-string sector will survive in the spectrum of unoriented string theories, as well as the graviton and the dilaton. Furthermore, in the sum over all worldsheets of closed and open strings, nonorientable surfaces such as the Möbius strip have to be included. 


\section{6}

\section{Superstring Theory}

The bosonic string theory is unsatisfactory in two respects. Since we observe fermions in nature, these particles should not be excluded in string theory. Moreover, the bosonic string theory is inconsistent because tachyons occur in the closed-string spectrum. This indicates an severe instability of the theory.

Remarkably, both problems can be solved by incorporating supersymmetry into string theory. There are two different approaches to superstring theory ${ }^{5}$ :

- The Green-Schwarz (GS) formalism is supersymmetric in ten-dimensional Minkowski spacetime, and can be generalized to curved background geometries with fluxes.

- The Ramond-Neveu-Schwarz (RNS) formalism is supersymmetric on the world-sheet of the fundamental string.

These approaches are equivalent at least in ten-dimensional Minkowski spacetime.

In this section the RNS approach to superstring theory is explained. In Section 1.6.1 we discuss the action for superstring theory. Moreover, we realize that the action is invariant under a supersymmetry transformation on the world-sheet. In Section 1.6.2 the possible boundary conditions for fermions are mentioned, which give rise to two different sectors: the Ramond and the Neveu-Schwarz (NS) sector. We see that the string theory is only consistent after applying a so-called GSOprojection. In Sections 1.6.3-1.6.5 the five consistent superstring theories in ten spacetime dimensions are discussed. The spectrum of massless fields is summarized in Table 1.1.

\subsection{1}

\section{The RNS Formalism of Superstring Theory}

The Polyakov action of the bosonic string in $D$-dimensional Minkowski spacetime reads, in the conformal gauge $h_{\alpha \beta}=\mathrm{e}^{\omega(\tau, \sigma)} \eta_{\alpha \beta}$,

$$
S=-\frac{1}{4 \pi \alpha^{\prime}} \int \mathrm{d}^{2} \sigma \partial_{\alpha} X_{\mu} \partial^{\alpha} X^{\mu}
$$

This action is supplemented by Virasoro constraints (1.67) and (1.68). For a supersymmetric world-sheet action, we have to introduce $D$ Majorana fermions $\psi^{\mu}$ transforming in the vector representation of the Lorentz group $S O(D-1,1)$. We therefore consider the Polyakov action supplemented by the usual Dirac action for $D$ free massless fermions,

$$
S=-\frac{1}{4 \pi \alpha^{\prime}} \int \mathrm{d}^{2} \sigma\left(\partial_{\alpha} X_{\mu} \partial^{\alpha} X^{\mu}+\mathrm{i} \bar{\psi}^{\mu} \gamma^{\alpha} \partial_{\alpha} \psi_{\mu}\right) .
$$

5) Recently, various approaches using spinor formalism were suggested by Berkovits. For a review see [20]. 
Here, $\gamma^{\alpha}$ are two-dimensional Dirac matrices satisfying the anticommutation relations $\left\{\gamma^{\alpha}, \gamma^{\beta}\right\}=2 \eta^{\alpha \beta} \mathbb{1}$. A convenient basis is

$$
\gamma^{0}=\left(\begin{array}{cc}
0 & -1 \\
1 & 0
\end{array}\right) \quad \text { and } \gamma^{1}=\left(\begin{array}{cc}
0 & 1 \\
1 & 0
\end{array}\right) .
$$

The world-sheet fields $\psi^{\mu}$ are Grassmann numbers consisting of two components

$$
\psi^{\mu}=\left(\begin{array}{c}
\psi_{-}^{\mu} \\
\psi_{+}^{\mu}
\end{array}\right),
$$

where $\psi_{-}^{\mu}$ and $\psi_{+}^{\mu}$ are real. In this notation, the fermionic part of the action takes the form

$$
S_{\mathrm{f}}=\frac{\mathrm{i}}{2 \pi \alpha^{\prime}} \int \mathrm{d}^{2} \sigma\left(\psi_{-}^{\mu} \partial_{+} \psi_{-\mu}+\psi_{+}^{\mu} \partial_{-} \psi_{+\mu}\right)
$$

with $\partial_{-}=\partial / \partial \sigma^{-}, \partial_{+}=\partial / \partial \sigma^{+}$and $\sigma^{ \pm}=\tau \pm \sigma$. The equations of motion are $\partial_{+} \psi_{-}^{\mu}=$ $\partial_{-} \psi_{+}^{\mu}=0$, which describe left- and right-moving waves. The action is invariant under the infinitesimal transformations

$$
\begin{aligned}
& \delta_{\varepsilon} X^{\mu}=\bar{\varepsilon} \psi^{\mu}, \\
& \delta_{\varepsilon} \psi^{\mu}=\gamma^{\alpha} \partial_{\alpha} X^{\mu} \varepsilon,
\end{aligned}
$$

where $\varepsilon$ is a constant infinitesimal Majorana spinor. This transformation mixes bosonic and fermionic world-sheet fields and is therefore a global supersymmetry transformation. Unfortunately, the supersymmetry algebra closes only on-shell, that is when the equations of motion are imposed. However, closure of the algebra can be achieved by introducing auxiliary fields. Moreover, we used the world-sheet theory in conformal gauge. There is a more fundamental formulation in which the world-sheet supersymmetry is a local symmetry. For details see [5].

\section{6 .2}

\section{Boundary Conditions for Fermions}

Next we consider the boundary conditions that arise from the superstring action. The possible boundary conditions of $X^{\mu}$ are discussed in Section 1.5.1. The boundary condition for the fermionic part reads

$$
\delta S_{\mathrm{f}}=-\frac{\mathrm{i}}{4 \pi \alpha^{\prime}} \int \mathrm{d} \tau\left[\psi_{+}^{\mu} \delta \psi_{+\mu}-\psi_{-}^{\mu} \delta \psi_{-\mu}\right]_{\sigma=0}^{\sigma=\pi} .
$$

\subsubsection{1}

\section{Open Strings}

For open strings we have to demand that the two terms for $\sigma=0$ and $\sigma=\pi$ vanish independently, that is

$$
\psi_{+}^{\mu} \delta \psi_{+\mu}-\psi_{-}^{\mu} \delta \psi_{-\mu}=0 \quad \text { for } \quad \sigma=0, \pi .
$$

Note that this is equivalent to

$$
\delta\left(\psi_{+\mu}\right)^{2}=\delta\left(\psi_{-\mu}\right)^{2} \quad \text { for } \quad \sigma=0, \pi .
$$


Since the overall sign of the components can be chosen arbitrarily, we demand $\psi_{+}^{\mu}(\tau, 0)=\psi_{-}^{\mu}(\tau, 0)$. If we want to impose the boundary conditions at $\sigma=\pi$ we have two options corresponding to the Ramond (R) sector and the Neveu-Schwarz (NS)-Sector of the theory,

$$
\begin{aligned}
\text { R: } \psi_{+}^{\mu}(\tau, \pi) & =+\psi_{-}^{\mu}(\tau, \pi), \\
\text { NS: } \psi_{+}^{\mu}(\tau, \pi) & =-\psi_{-}^{\mu}(\tau, \pi) .
\end{aligned}
$$

The mode decomposition in the R and NS sector is given by

$$
\begin{aligned}
\mathrm{R}: \psi_{\mp}^{\mu}(\tau, \sigma) & =\frac{1}{\sqrt{2}} \sum_{n \in \mathbb{Z}} d_{n}^{\mu} \mathrm{e}^{-\mathrm{i} n \sigma_{\mp}}, \\
\mathrm{NS}: \psi_{\mp}^{\mu}(\tau, \sigma) & =\frac{1}{\sqrt{2}} \sum_{r \in \mathbb{Z}+\frac{1}{2}} b_{r}^{\mu} \mathrm{e}^{-\mathrm{i} r \sigma_{\mp}},
\end{aligned}
$$

where $d_{n}^{\mu}$ and $b_{r}^{\mu}$ are Grassmann numbers.

The string states are constructed by acting on the ground state of the NS and R sector with creation operators. The ground state in the NS sector $|0\rangle_{\mathrm{NS}}$ is a spacetime boson and therefore all string states in this sector are bosonic in spacetime since the oscillators act as vectors in spacetime. Furthermore, the ground state of the NS sector is tachyonic and will be removed. The first excited string state, which is generated by applying a creation operator to the ground state of the NS sector, is a massless vector boson.

By contrast, the ground states in the $\mathrm{R}$ sector, which are massless spacetime fermions, are degenerate and differ by chirality in spacetime. By applying creation operators to the ground state of the NS sector, massive string states are obtained. Moreover, all states of the $\mathrm{R}$ sector are spacetime fermions.

The spectrum of the NS and R sector can be truncated in a specific way which eliminates the tachyon. This truncation is called GSO projection, named after Gliozzi, Scherk, and Olive [21]. The GSO projection also ensures that the partition function on the two-torus is modular invariant. In the NS sector only states with an odd number of creation operators $b_{-r}^{\mu}, r>0$ applied to the ground state $|0\rangle_{\mathrm{NS}}$ are kept in the spectrum. The GSO projection leaves an equal number of bosons and fermions at each mass level, as required by spacetime supersymmetry. At the massless level the states of the NS sector are massless gauge bosons, whereas the R sector includes the supersymmetric partner of the gauge boson, the gaugino.

\subsubsection{2}

\section{Closed String}

As we saw in bosonic string theory, a closed string consists essentially of left- and right-moving copies of an open string. Since an open superstring has two different sectors (NS and R), the closed-string sector can be constructed in four ways by combining the left-moving sector (NS and R) and the right-moving one (NS and R). The NS-NS and R-R states are spacetime bosons, whereas the NS-R and R-NS states 
are spacetime fermions. Applying a GSO projection as in the open superstring case leads to a supersymmetric theory in spacetime.

The NS-NS sector of oriented strings includes at the massless level exactly the same states as the closed sector of the oriented bosonic string theory: the graviton $g_{\mu \nu}$, the Kalb-Ramond field $B_{\mu \nu}$, and the dilaton $\phi$. The NS-R and R-NS states, which are fermionic in spacetime, contain the gravitino, the supersymmetric partner of the graviton, and the dilatino, the supersymmetric version of the dilaton. The story for the R-R sector is a little more subtle due to the degeneracy of ground states of the R-sector. We will see that two different superstring theories are obtained: type IIA and type IIB.

\subsection{3}

\section{Type IIA and Type IIB Superstring}

Since the R-sector has two possible inequivalent ground states, which differ by chirality, we can choose ground states with the same chirality for the left- and rightmoving sector. This corresponds to type IIB superstring theory. The R-R sector consists of a scalar field $C_{(0)}$, an antisymmetric field $C_{(2)}$, and a totally antisymmetric rank-four tensor field $C_{(4)}$ at the massless level. ${ }^{6}$ If the $\mathrm{R}$ sector ground states for the left- and right-moving modes have different chiralities, we are led to type IIA superstring theory. In the type IIA theory the massless R-R bosons are given by a gauge field $C_{(1)}$ and a totally antisymmetric rank-three tensor field $C_{(3)}{ }^{\text {7) }}$

Although type IIA and type IIB superstring theories are inequivalent, there exist dualities between both theories. In addition to the type II theories, there are three other consistent superstring theories in ten dimensions: type I superstring theory and two heterotic string theories.

\subsection{4}

\section{Type I Superstring}

Type I superstring theory can be constructed as a projection of type IIB superstring theory. Since in type IIB the superstrings are oriented, the world-sheet parity transformation $\Omega$ may be gauged, which exchanges the left- and right-moving modes of the world-sheet fields $X^{\mu}$ and $\psi^{\mu}$. This is a symmetry of type IIB string theory. Therefore, the string states may be consistently truncated and we may consider only those which are even under the world-sheet parity transformation. Consequently, type I superstring theory contains unoriented superstrings. When the projection described is imposed, the massless bosonic closed-string states are the graviton and the dilaton of the NS-NS sector as well as the antisymmetric $C_{(2)}$ of the R-R sector. In contrast to type II superstring theories, it is necessary to add a twisted sector to

6) The components of $C_{(0)}, C_{(2)}$, and $C_{(4)}$ are denoted by $C, C_{\mu \nu}$ and $C_{\mu v o \sigma}$, i.e. the number of indices is not explicitly specified for the components.
7) The components of $C_{(1)}$ and $C_{(3)}$ are called $C_{\mu}$ and $C_{\mu \nu \varrho}$, respectively. 
the closed-string sector, which are the type I open strings. These open strings give rise to a gauge theory with gauge group $S O(32)$. For details and further references see [4] or [5].

\subsection{5}

\section{Heterotic Superstring}

Besides type I and type II superstring theories, there are also two heterotic superstring theories. These are constructed as follows.

In closed-string theories, left- and right-moving modes are essentially independent ${ }^{8}$. This enables us in type II string theories to create string states for which the right-moving modes are in the NS sector and the left-moving modes are in the $\mathrm{R}$ sector. Actually a much more drastic asymmetry between the left and right-moving modes may be introduced. It turns out that it is possible to make the left-moving modes supersymmetric by using a copy of the open superstring and combine them with right-moving modes of a bosonic open string.

We will now discuss the fermionic construction of the heterotic string. First of all let us consider the standard superstring action where the right-moving fermionic coordinates $\psi_{+}^{\mu}$ are omitted,

$$
S=-\frac{1}{4 \pi \alpha^{\prime}} \int \mathrm{d}^{2} \sigma\left(\partial_{\alpha} X^{\mu} \partial^{\alpha} X_{\mu}-2 \mathrm{i} \psi_{-}^{\mu} \partial_{+} \psi_{\mu-}\right) .
$$

As in the superstring case, the left-moving sector of this theory is consistent in ten dimensions only. Since the right-moving sector consists of ten bosonic coordinates (and not of 26), we have to add additional right-moving fields to the action. In order to avoid adding new left-moving modes at the same time, we have to add fermionic right-moving fields only. If these fermionic fields carry a vector index in spacetime, we are back to the standard superstring action. Therefore, we will instead demand that the right-moving fermionic fields are scalars in spacetime. For consistency we need 32 of them,

$$
S=-\frac{1}{4 \pi \alpha^{\prime}} \int \mathrm{d}^{2} \sigma\left(\partial_{\alpha} X^{\mu} \partial^{\alpha} X_{\mu}-2 \mathrm{i} \psi_{-}^{\mu} \partial_{+} \psi_{\mu-}-2 \mathrm{i} \lambda_{+}^{A} \partial_{-} \lambda_{+}^{A}\right),
$$

where $A=0, \ldots, 31$. The action has an $S O(32)$ world-sheet symmetry, under which the $\lambda^{A}$ transform in the fundamental transformation. This global world-sheet symmetry gives rise to a local gauge symmetry of the spacetime theory. The only possible gauge groups depend on the boundary conditions for $\lambda^{A}$ and on the GSO projection as well as on the cancelation of certain anomalies. There are two different inequivalent heterotic string theories: $S O(32)$ heterotic string theory and $E_{8} \times E_{8}$ heterotic string theory.

The heterotic string theories are an extremely attractive starting point for the construction of standard model-like theories. This is due to the fact that the heterotic

8) The left and right-moving modes are coupled

only by the level-matching condition (see

Section 1.5.2 for the bosonic string). 
Table 1.1 Bosonic massless fields of the five consistent superstring theories in ten spacetime dimensions. $g_{\mu \nu}$ and $\phi$ are the metric and the dilaton, respectively. The KalbRamond field is denoted by $B_{(2)}$. Moreover, in superstring theories there exist $p$-form gauge potentials $C_{(p)}$ in the R-R sector of the closed string. In heterotic and type I superstring theories there are also non-Abelian gauge degrees of freedom $A_{\mu}^{a}$ present. The corresponding gauge groups are listed in the last column. By adding $D$-branes to string theories other gauge groups are possible.

\begin{tabular}{llll}
\hline Type & $\begin{array}{l}\text { Number of } \\
\text { supercharges }\end{array}$ & Bosonic massless fields & Non-Abe \\
& & & \\
\hline Heterotic $S O(32)$ & 16 & $g_{\mu \nu}, \phi, B_{(2)}, A_{\mu}^{a}$ & $S O(32)$ \\
Heterotic $E_{8} \times E_{8}$ & 16 & $g_{\mu \nu}, \phi, B_{(2)}, A_{\mu}^{a}$ & $E_{8} \times E_{8}$ \\
IIA & $16+\overline{16}$ & $g_{\mu \nu}, \phi, B_{(2)}, C_{(1)}, C_{(3)}$ & - \\
IIB & $16+16$ & $g_{\mu \nu}, \phi, B_{(2)}, C_{(0)}, C_{(2)}, C_{(4)}$ & - \\
I & 16 & $g_{\mu \nu}, \phi, A_{\mu}^{a}, C_{(2)}$ & $S O(32)$
\end{tabular}

string theories are chiral, and have fermions and non-Abelian gauge symmetries automatically built in. During the first String Revolution, many realistic features of the standard model were found in the $E_{8} \times E_{8}$ heterotic string. This is due to the fact that GUT (Grand Unified Theory) gauge groups are contained in $E_{6}$ and therefore also in $E_{8}$.

Altogether, there are five consistent superstring theories in ten spacetime dimensions: type I, type IIA, and type IIB as well as $S O(32)$ heterotic and $E_{8} \times E_{8}$ heterotic string theory. Although all these theories describe vibrating strings, the details are quite different.

1.7

\section{String Dualities and M-Theory}

During the second string revolution, which took place in the mid 1990s, it was realized that all five consistent superstring theories in ten dimensions are related to each other by a complicated web of dualities. This will be presented in this section. In particular, the low-energy dynamics of superstring theories will be discussed. Furthermore, two novel dualities between string theories are introduced: T-Duality and S-Duality.

In addition there is evidence that all superstring theories are related to an elevendimensional theory, called M-theory. An introduction to M-theory is given at the end of the section.

\subsection{1}

\section{Low-Energy Effective Action of Superstring Theory}

As in the bosonic case (see Section 1.5.4) we can write down a spacetime action taking into account the effects of the massless superstring excitations. These exci- 
tations are listed in table 1.1. Since the effective supersymmetric action necessarily incorporates gravity ${ }^{\text {") }}$, the theory is called supergravity.

The low-energy effective action for type IIA and type IIB superstring theories are called type IIA and type IIB supergravity, respectively. As an example, we consider the action of type IIB supergravity. Type IIB superstring theory consists of the following closed-string states at the massless level: the metric $g_{\mu v}$, the NS-NS KalbRamond field $B_{\mu \nu}$, the dilaton $\phi$ as well as the $p$-form R-R potentials $C_{(0)}, C_{(2)}$, and $C_{(4)}$. Moreover, we define as linear combinations of these fields the axion-dilaton scalar $\tau$ as well as the complex three-form $G_{(3)}$ by

$$
\tau=C_{(0)}+\mathrm{ie}^{-\phi}, \quad G_{(3)}=F_{(3)}-\tau H_{(3)} .
$$

Here, $F_{(3)}$ and $H_{(3)}$ are the field strength of $C_{(2)}$ and $B_{(2)}$, that is $F_{(3)}=\mathrm{d} C_{(2)}$ and $H_{(3)}=\mathrm{d} B_{(2)}$. The field strength of $C_{(4)}$ is given by $F_{(5)}=\mathrm{d} C_{(4)}$. More important is the self-dual combination

$$
\tilde{F}_{(5)} \equiv F_{(5)}+\frac{1}{2} B_{(2)} \wedge F_{(3)}-\frac{1}{2} C_{(2)} \wedge H_{(3)} .
$$

The type IIB supergravity action in the Einstein frame then reads

$$
\begin{aligned}
S_{\text {IIB }}= & \frac{1}{2 \kappa_{10}^{2}} \int \mathrm{d}^{10} x \sqrt{-\mathrm{g}}\left[R-\frac{\left|\partial_{\mu} \tau\right|^{2}}{2(\operatorname{Im} \tau)^{2}}-\frac{\left|G_{(3)}\right|^{2}}{12 \operatorname{Im} \tau}-\frac{\left|\tilde{F}_{(5)}\right|^{2}}{4 \cdot 5 !}\right\} \\
& +\frac{1}{8 \mathrm{i} \kappa_{10}^{2}} \int \frac{C_{(4)} \wedge G_{(3)} \wedge \bar{G}_{(3)}}{\operatorname{Im} \tau},
\end{aligned}
$$

where the ten-dimensional gravitational coupling is $2 \kappa_{10}^{2}=16 \pi G_{10}=1 /(2 \pi)\left(2 \pi l_{\mathrm{s}}\right)^{8} \mathrm{~g}_{\mathrm{s}}^{2}$ and $l_{\mathrm{s}}=\sqrt{\alpha^{\prime}}$ is the string length. Moreover, we have to impose the self-duality constraint of $\tilde{F}_{(5)}$ at the level of the equations of motion by hand, that is $\star \tilde{F}_{(5)}=\tilde{F}_{(5)}$, where $\star$ denotes the Hodge star operator.

\subsection{2}

\section{T-Duality}

T-Duality (or target space duality) denotes the equivalence between two superstring theories compactified on different background spacetimes. Let us consider bosonic string theory compactified on a circle, that is the coordinate $X^{25}$ is periodically identified in the following way,

$$
X^{25} \sim X^{25}+2 \pi R
$$

9) These effective spacetime theories are not only invariant under global supersymmetry transformations, but also under local ones. Since the commutator of two supersymmetry transformations is a translation, the theory is also invariant under local diffeomorphisms and therefore contains gravity. 


\subsubsection{1}

\section{T-Duality of Closed Strings}

Now let us restrict ourselves to closed strings. The embedding function $X^{25}(\tau, \sigma)$ has to satisfy the periodicity condition

$$
X^{25}(\tau, \sigma+2 \pi)=X^{25}(\tau, \sigma)+2 m \pi R,
$$

where $R$ is the radius of the circle and $m$ is an arbitrary integer. The number $m$ counts how often the closed string winds around the compactified direction $X^{25}$ and is therefore called the winding number. In the noncompactified directions, the mode decomposition (1.71) and (1.72) for the right and left-moving modes can be used subject to $p_{\mathrm{R}}^{\mu}=p_{\mathrm{L}}^{\mu}$. In the compactified direction the same mode decomposition can be applied, however now with $p_{\mathrm{R}}^{25} \neq p_{\mathrm{L}}^{25}$. Omitting the oscillatory terms, we have the decomposition

$$
\begin{aligned}
X_{\mathrm{R}}^{25}(\tau-\sigma) & =\frac{1}{2} x_{0}^{\mu}+\alpha^{\prime} p_{\mathrm{R}}^{\mu}(\tau-\sigma)+\ldots \\
X_{\mathrm{L}}^{25}(\tau+\sigma) & =\frac{1}{2} x_{0}^{\mu}+\alpha^{\prime} p_{\mathrm{L}}^{\mu}(\tau+\sigma)+\ldots
\end{aligned}
$$

Since $X^{25}=X_{\mathrm{L}}^{25}+X_{\mathrm{R}}^{25}$, the periodicity condition reads

$$
\alpha^{\prime}\left(p_{\mathrm{L}}^{25}-p_{\mathrm{R}}^{25}\right)=m R .
$$

Since the $X^{25}$ direction is compactified, the center-of-mass momentum $p_{\mathrm{R}}^{25}+p_{\mathrm{L}}^{25}$ is quantized in units of $1 / R$, that is

$$
p_{\mathrm{L}}^{25}+p_{\mathrm{R}}^{25}=\frac{n}{R} .
$$

Thus, $p_{\mathrm{R}}^{25}$ and $p_{\mathrm{L}}^{25}$ are given by

$$
\begin{aligned}
& p_{\mathrm{L}}^{25}=\frac{1}{2}\left(\frac{n}{R}+\frac{m R}{\alpha^{\prime}}\right), \\
& p_{\mathrm{R}}^{25}=\frac{1}{2}\left(\frac{n}{R}-\frac{m R}{\alpha^{\prime}}\right) .
\end{aligned}
$$

We are now interested in the spectrum of the closed-string states. First of all, the level-matching condition (see Section 1.5.2) for the closed string is modified,

$$
\bar{N}-N=n m,
$$

and the mass formula for string states reads

$$
M^{2}=\left(\frac{m R}{\alpha^{\prime}}\right)^{2}+\left(\frac{n}{R}\right)^{2}+\frac{2}{\alpha^{\prime}}(N+\bar{N}-2) .
$$

However, this is not the whole story. The closed-string sector has a remarkable symmetry. Considering the mass formula, it turns out that the closed-string spectrum for a compactification with radius $R$ is identical to the closed-string spectrum for 
a compactification with radius $\tilde{R}=\alpha^{\prime} / R$ if we interchange the winding number $m$ and momentum number $n$,

$$
\begin{aligned}
R & \leftrightarrow \tilde{R}=\frac{\alpha^{\prime}}{R}, \\
(n, m) & \leftrightarrow(m, n) .
\end{aligned}
$$

Although here we have described the proof for T-duality only for free strings, it can be shown that T-duality of closed strings is an exact symmetry at the quantum level also if interactions are included.

In fact it is not possible to distinguish between both compactifications. Note that if $R$ is large, then the dual radius $\widetilde{R}$ is small. This is a remarkable feature, which is not present in usual field theories of point-like particles. Since T-duality exchanges the winding number on the circle with the quantum number of the corresponding (discrete) momentum, it is clear that this symmetry has no counterpart in ordinary point particle field theory, as the ability of closed strings to wind around the compact dimension is essential.

\subsubsection{2}

\section{T-Duality of Open Strings}

At first sight, it seems that T-duality does not apply to theories with open strings, since open strings do not have a winding sector. However, this is only apparently so [22]. T-duality can be restored in the open-string sector with the help of $D$-branes which are hyperplanes where open strings end. By applying T-duality, not only the radius of the compactified dimension changes, but also the dimension of the $D$ brane.

To see this, let us consider the propagation of open bosonic strings in a spacetime which is compactified in the $X^{25}$ direction. Furthermore, we assume for simplicity that we have a space-filling D25-brane, that is the endpoints of the string can move freely. As it was in the case of closed strings, the center-of-mass momentum in the compactified direction is quantized, that is $p^{25}=n / R$ and contributes terms of the form $n^{2} / R^{2}$ to the mass formula of string states. However, this contribution changes if we apply the T-duality rules of closed strings only. Since the dual radius is $\tilde{R}=\alpha^{\prime} / R$, the contribution to the mass formula changes to $n^{2} \widetilde{R}^{2} / \alpha^{\prime 2}$.

T-duality can be restored in the open-string sector by considering $D$-branes. Instead of the D25-brane described above, consider now a D24-brane in the dual theory, which does not wrap the $X^{25}$-direction. Because of the Dirichlet boundary conditions, we have no momentum states in the compact direction. In addition, the endpoints of the open string must remain attached to points with $x^{25}=x_{0}^{25}+2 \pi n \tilde{R}$, where $x_{0}^{25}$ is the position of the D24-brane in the compactified direction. Therefore, we get winding states in the dual theory which contribute to the mass formula by

$$
\left(\frac{n \tilde{R}}{\alpha^{\prime}}\right)^{2}=\left(\frac{n}{R}\right)^{2} .
$$

This is precisely the contribution of the momentum states in the original theory with a space-filling D25-brane. 
Therefore, T-duality is an exact symmetry of the open-string sector, if the dimension of the $D$-brane is also changed. This means that the type of boundary conditions of open strings (Neumann or Dirichlet) has to be exchanged in the direction in which T-duality is performed.

As an example consider a $D 24$-brane stretched along the coordinates $X^{0}, X^{1}, \ldots$, $X^{24}$. In these directions Neumann boundary conditions for open strings are imposed. Moreover, in the $X^{25}$-direction open strings will satisfy Dirichlet boundary conditions. Assuming that the $X^{24}$ and $X^{25}$ directions are compactified on circles with radius $R_{24}$ and $R_{25}$, respectively, we can apply T-duality to both compact directions. If we perform a T-duality along $X^{25}$, the open strings in the dual theory in the $X^{25}$-direction obey also Neumann boundary conditions. Therefore, in the dual theory a D25-brane exists and the radii of the two compactified directions are given by $R_{24}$ and $\alpha^{\prime} / R_{25}$, respectively. If we apply a T-duality along $X^{24}$ instead, the open strings no longer satisfy Neumann boundary conditions in the $X^{24}$-coordinate. Therefore, we are left with a D23-brane in the dual theory, which is compactified on circles with radii $\alpha^{\prime} / R_{24}$ and $R_{25}$.

\subsubsection{3}

\section{T-Duality in Superstring Theory}

Up to now we have only discussed the rules of T-duality in bosonic string theory. However, T-duality is also an exact symmetry of superstring theories. In fact TDuality relates the following superstring theories:

- Heterotic $S O(32)$ superstring theory on a circle with radius $R$ and heterotic $E_{8} \times E_{8}$ superstring theory on a circle with radius $\alpha^{\prime} / R$.

- Type IIA superstring theory on a circle with radius $R$ and type IIB superstring theory on a circle with radius $\alpha^{\prime} / R$.

\subsection{3}

\section{S-Duality}

S-duality is a strong-weak coupling duality, in the sense that a superstring theory in the weak coupling regime is mapped to another strongly coupled superstring theory. S-duality relates the string coupling constant $g_{\mathrm{s}}$ to $1 / g_{\mathrm{s}}$ in the same way that T-duality maps the radii of the compactified dimension $R$ to $\alpha^{\prime} / R$.

The most prominent example where S-duality is present is type IIB superstring theory. This theory is mapped to itself under S-duality. This is due to the fact that S-duality is a special case of the $S L(2, \mathbb{Z})$ symmetry of type IIB superstring theory: in the massless spectrum of type IIB superstring theory, the scalars $\phi$ and $C_{(0)}$ and the two-form potentials $B_{(2)}$ and $C_{(2)}$ are present in pairs. Arranging the R-R scalar $C_{(0)}$ and the dilaton $\phi$ in a complex scalar $\tau=C_{(0)}+i \exp (-\phi)$, the $S L(2, \mathbb{R})$ symmetry of the equations of motion of type IIB supergravity (see (1.117)) acts as

$$
\tau \rightarrow \frac{a \tau+b}{c \tau+d}
$$


with the real parameter $a, b, c, d$ satisfying $a d-b c=1$. Moreover, the R-R two-form potential $C_{(2)}$ and the NS-NS $B_{(2)}$ transform according to

$$
\left(\begin{array}{l}
B_{(2)} \\
C_{(2)}
\end{array}\right) \rightarrow\left(\begin{array}{cc}
d & -c \\
-b & a
\end{array}\right)\left(\begin{array}{l}
B_{(2)} \\
C_{(2)}
\end{array}\right) .
$$

Because of charge quantization, this symmetry group breaks down to $S L(2, \mathbb{Z})$ of the full superstring theory. A particular case of the above symmetry is S-duality. If the R-R scalar $C_{(0)}$ vanishes, the coupling constant $g_{s}=\exp (\phi)$ of type IIB superstring theory can be mapped to $1 / g_{\mathrm{s}}$ by the $S L(2, \mathbb{Z})$ transformation with $a=d=0$, and $b=-c=1$, that is

$$
\phi \rightarrow-\phi, \quad B_{(2)} \rightarrow C_{(2)}, \quad C_{(2)} \rightarrow-B_{(2)} .
$$

Note that the $S L(2, \mathbb{Z})$ duality of type IIB superstring theory is a strong-weak coupling duality relating different regimes of the same theory.

Since the NS-NS field $B_{(2)}$ couples to the fundamental string, the fundamental string carries one unit of $B_{(2)}$ charge, but is not charged under the NS-NS twoform field $C_{(2)}$. However, there are also solitonic strings which are charged under the NS-NS two-form field $C_{(2)}$, but not under the Kalb-Ramond field $B_{(2)}$. These objects are D1-branes (see also Section 1.8). Under S-duality, a fundamental string is transformed into a $D 1$-brane and vice versa. Moreover, a general $S L(2, \mathbb{Z})$ transformation maps the fundamental string into a bound state $(p, q)$, carrying $p$ units of NS-NS charge and $q$ units of R-R charge.

\subsection{4}

\section{Web of Dualities and M-Theory}

So far we have seen that type IIA and type IIB superstring theory are related by T-duality. The same applies to the two heterotic string theories. Moreover, we also discussed S-duality in type IIB superstring theory. In contrast to T-duality, S-duality is a strong-weak coupling duality $g_{\mathrm{s}} \rightarrow 1 / g_{\mathrm{s}}$, and therefore the role of fundamental strings and $D 1$-branes are exchanged. In the strong coupling limit, $D 1$-branes are now the fundamental degrees of freedom since their tension $\tau_{D 1}=\left(2 \pi \alpha^{\prime} \mathrm{g}_{\mathrm{s}}\right)^{-1}$ is smaller than the tension $\tau=\left(2 \pi \alpha^{\prime}\right)^{-1}$ of the fundamental string. Another example for S-duality are heterotic $S O(32)$ and type I superstring theory. To be more precise: the strong coupling limit of type I superstring theory is weakly coupled $S O(32)$ heterotic string theory.

S-duality and T-duality are embedded into a larger symmetry group, $U$-duality [23]. By using this U-duality, it was realized in 1995 that the strong coupling regime of all five consistent string theories in ten spacetime dimensions is mapped to some weakly coupled limit of another theory ([24], for a review see [25]). For this picture to be self-contained, one has to include also eleven-dimensional supergravity. It is believed that all five consistent string theories can be unified into an elevendimensional parent theory, called $M$-theory. Although up to now a precise definition 
of M-theory is not available, we know that the low-energy effective action of this theory is given by the unique eleven-dimensional supergravity.

At strong coupling fundamental strings are no longer the fundamental degrees of freedom of superstring theory. Therefore, we can not expect that only tendimensional target spacetimes are consistent since this was derived by considering the trace of the energy-momentum tensor of the world-sheet of fundamental strings.

\subsubsection{1}

\section{Type IIA String Theory and M-Theory}

Let us explore the connection between type IIA superstring theory and M-theory in more detail. The first evidence of such a connection was found by [26]: type IIA supergravity, which is the low-energy limit of type IIA superstring theory, can be derived from a Kaluza-Klein reduction of eleven-dimensional supergravity. The bosonic spectrum of eleven-dimensional supergravity consists of a metric $G_{\mathrm{MN}}$ and a three-form potential $A_{(3)}$ with components $A_{\text {MNP. }}$. A Kaluza-Klein reduction to $D=10$ is performed by putting the eleventh coordinate $X^{10}$ on a circle of radius $R_{10}$. Decomposing the fields with respect to the ten-dimensional Lorentz group, we can identify the field content of type IIA supergravity. The metric $G_{\mathrm{MN}}$ and threeform potential $A_{(3)}$ of eleven-dimensional supergravity is related to the dilaton $\phi$, to the metric $g_{\mu \nu}$, and to the R-R-potentials $C_{(1)}$ and $C_{(3)}$ in the following schematic way, symbolized by $\sim$ :

$$
\begin{aligned}
G_{\mathrm{MN}} \rightarrow & \phi \sim G_{1010}, \\
& C_{\mu} \sim G_{\mu 10}, \\
& g_{\mu \nu} \sim G_{\mu v}, \\
A_{\mathrm{MNP}} \rightarrow & B_{\mu v} \sim A_{\mu v 10}, \\
& C_{\mu v \varrho} \sim A_{\mu v \varrho},
\end{aligned}
$$

where $\mu, v, \varrho=0, \ldots, 9$. Moreover, the ten-dimensional fields are independent of the eleventh, internal coordinate $X_{10}$. To be precise, the metric of the elevendimensional spacetime is determined by the field content of type IIA superstring theory

$$
d s^{2}=G_{M N} d x^{M} d x^{N}=\exp (-2 / 3 \phi) g_{\mu \nu} d x^{\mu} d x^{\nu}+\exp (4 / 3 \phi)\left(d x^{11}+C_{\mu} d x^{\mu}\right)^{2} .
$$

Therefore, we see that a distance measured in ten-dimensional spacetime is multiplied by $g_{\mathrm{s}}^{-1 / 3}$ in eleven-dimensional spacetime. The Planck length $l_{\mathrm{p}}$ is determined by the string length $l_{\mathrm{s}}=\sqrt{\alpha^{\prime}}$,

$$
l_{\mathrm{p}}=\mathrm{g}_{\mathrm{s}}^{1 / 3} l_{\mathrm{s}} .
$$

Integrating out the eleventh dimension yields a prefactor in front of the action, relating the ten- and eleven-dimensional gravitational coupling constants $\kappa_{10}$ and 
$\kappa_{11}$ by

$$
\kappa_{10}^{2}=\frac{\kappa_{11}^{2}}{2 \pi R_{10}} .
$$

Using the eleven-dimensional gravitational constant

$$
16 \pi G_{11}=2 \kappa_{11}^{2}=\frac{1}{2 \pi}\left(2 \pi l_{\mathrm{p}}\right)^{9},
$$

we can deduce the radius $R_{10}$ of the compactified direction,

$$
R_{10}=\mathrm{g}_{\mathrm{s}}^{2 / 3} l_{\mathrm{p}}=\mathrm{g}_{\mathrm{s}} l_{\mathrm{s}}=\mathrm{g}_{\mathrm{s}} \sqrt{\alpha^{\prime}} .
$$

For small string coupling constant $g_{\mathrm{s}}$ the radius $R_{10}$ of the compactified direction in units of the string length is small and the spacetime is effectively ten-dimensional. However, for large string coupling constants the spacetime is eleven-dimensional.

As discussed in the last paragraph, the low-energy limit of type IIA superstring theory, which is given by type IIA supergravity, can be obtained by a Kaluza-Klein reduction of eleven-dimensional supergravity on a circle $S^{1}$. However, the relation between type IIA supergravity and M-theory is much deeper. Let us match nonperturbative objects on both sides. In the nonperturbative part of its spectrum elevendimensional supergravity has $M 2$ - and M5-branes, which are charged under the four-form field strength $G_{(4)}=\mathrm{d} A_{(3)}$. The nonperturbative spectrum of type IIA supergravity contains stable $D p$-branes with $p$ even as well as NS5-branes.

Let us consider some examples to see how $D$-branes and $M$-branes are related. The D0-branes of type IIA are mapped to Kaluza-Klein of the massless supergravity multiplet. An evidence for this statement is the mass of the two states. Since $D 0$-branes are nonperturbative, their mass is proportional to $\mathrm{g}_{\mathrm{s}}^{-1}$. A bound state of $n$ D0-branes therefore has mass $\sim n / g_{s}$. This matches precisely with mass states of Kaluza-Klein excitations on a circle with radius $g_{s}$ in units of the string length. Moreover, the fundamental string and D2-branes can be related to M2-branes by dimensional reduction of $x^{10}:$ in the case of the fundamental string the M2 brane is wrapped on the compactified coordinate $x^{10}$. Performing a dimensional reduction we obtain a fundamental string. If the $M 2$ brane is not wrapped along the compactified dimension we get a D2-brane. In addition, D4 and NS5 branes can be mapped to M5-branes of M-theory. For further details see [26].

In summary, we gave evidence that type IIA superstring theory is dual to Mtheory on $S^{1}$, where the radius of the circle in units of the string length is given by the string coupling constant $g_{s}$. This implies that the perturbative regime of string theory is ten-dimensional because the radius of the compactified coordinate is small. Moreover, in the nonperturbative regime, the radius becomes large and the coordinate is effectively decompactified.

\subsubsection{2}

\section{Heterotic $E_{8} \times E_{8}$ String Theory and M-Theory}

A similar picture arises in the strong coupling limit of the heterotic $E_{8} \times E_{8}$ string theory, which is equivalent to M-theory on the orbifold $S^{1} / \mathbb{Z}_{2}$ [27], that is we consider only string states which are even under the $\mathbb{Z}_{2}$-symmetry $x^{10} \rightarrow-x^{10}$ and 
$A_{(3)} \rightarrow-A_{(3)}$ of M-theory on $\mathbb{R}^{9,1} \times S^{1}$. The orbifold $S^{1} / \mathbb{Z}_{2}$, can be viewed as a line segment from $x^{10}=0$ to $x^{10}=\pi R_{10}$, where $R_{10}$ is related to the string coupling constant as in type IIA superstring theory. The super Yang-Mills fields are located at the fixed points of the orbifold, $x_{10}=0$ and $x_{10}=\pi R_{10}$. Therefore, these boundaries of the eleven-dimensional spacetime are called "end-of-the-world-9-branes". Each of the branes carry non-Abelian gauge fields with gauge group $E_{8}$. In this picture the product gauge group $E_{8} \times E_{8}$ can be understood. Moreover, the fundamental strings of heterotic $E_{8} \times E_{8}$ string theory are precisely the cylindrical M2-branes suspended between the "end-of-the-world-9-branes".

This picture of strongly coupled heterotic $E_{8} \times E_{8}$ string theory is the starting point of heterotic M-theory. By compactifying (usually on Calabi-Yau manifolds) to four spacetime dimensions it is possible to investigate phenomenologically interesting strong coupling effects. For more details see Chapter 8.

\section{8}

\section{D-Branes}

So far we have considered $D$-branes as hyperplanes where fundamental strings can end. This is a microscopic point-of-view, since $D$-branes are defined as boundaries of world-sheets of open strings. The massless string excitations of open strings ending on $D$-branes can be described in general by a supersymmetric (non-)Abelian gauge theory. The precise form of the effective action is discussed in Section 1.8.1.

In addition, $D$-branes are also solitonic solutions to the macroscopic equations of motion for the low-energy theory of superstring theory, governed effectively by supergravity. In that macroscopic point-of-view $D$-branes are objects such as black holes, cosmic strings, monopoles, which curve the surrounding spacetime. These $D$-branes are a further ingredient to superstring theory besides fundamental strings. Moreover, BPS D-branes carry charges of the R-R $p$-form gauge fields $C_{(p)}$. Because of charge conservation BPS $D$-branes are stable. In type IIA/IIB superstring theory $D p$-branes with $p$ even/odd are BPS since in this superstring theory RR gauge potentials $C_{(p+1)}$ are present to which $D p$-branes couple [28]. However, unlike fundamental strings, $D$-branes are nonperturbative since the tension and therefore also the energy scales as $1 / g_{s}$. This aspect of $D$-branes is investigated in Section 1.8.2.

\subsection{1}

\section{Effective Action of $D$-Branes}

The dynamics of the massless open-string modes ending on one $D p$-brane (or on one anti- $D p$-brane) is described by an effective action

$$
S_{D p}=S_{\mathrm{DBI}} \pm S_{\mathrm{CS}}
$$

consisting of a Dirac-Born-Infeld (DBI) and a Chern-Simons (CS) action. The relative sign between the DBI and CS part of the action depends on whether it is 
a $D p$ or anti- $D p$-brane (also denoted by $\overline{D p}$-brane). The plus sign corresponds to a $D p$-brane.

The interaction of the NS-NS massless fields $g_{\mu \nu}, B_{\mu \nu}$, and $\phi$ as well as the NS field strength $F_{a b}$ on the $D p$-brane are given by the DBI action

$$
S_{\mathrm{DBI}}=-\mu_{\mathrm{p}} \int_{D p} \mathrm{~d}^{p+1} \zeta \mathrm{e}^{-\mathcal{P}[\phi]} \sqrt{-\operatorname{det}\left(\mathcal{P}[g]_{a b}+\mathcal{F}_{a b}\right)},
$$

where $\mathcal{F}_{a b}=2 \pi \alpha^{\prime} F_{a b}+\mathcal{P}[B]_{a b}$ and $\mathcal{P}[B]_{a b}, \mathcal{P}[g]_{a b}$ are the pullback of $B_{\mu \nu}$ and $g_{\mu \nu}$, that is

$$
\mathcal{P}[B]_{a b}=\partial_{a} X^{\mu} \partial_{b} X^{v} B_{\mu \nu}, \quad \mathcal{P}[g]_{a b}=\partial_{a} X^{\mu} \partial_{b} X^{v} g_{\mu \nu},
$$

with $\partial_{a}=\partial / \partial \xi^{a}$ and $a, b,=0, \ldots, p$. The constant $\mu_{\mathrm{p}}$ is given by $\mu_{\mathrm{p}}=(2 \pi)^{-p} \alpha^{\prime-(p+1) / 2}$ for type II theories.

Expanding the DBI action by using $\operatorname{det}(\mathbb{1}+M)=1-1 / 4 \operatorname{tr}\left(M^{2}\right)$ for antisymmetric matrices $M$ we see that the DBI action is a generalization of a Yang-Mills action

$$
S_{\mathrm{DBI}} \simeq \frac{\left(2 \pi \alpha^{\prime}\right)^{2}}{4} \int_{D p} \mathrm{~d}^{p+1} \zeta \mathcal{F}_{a b} \mathcal{F}^{a b} .
$$

Moreover, we consider only a constant dilaton field $\phi$. The $D$-brane tension is defined by $\tau_{\mathrm{p}}=\mu_{\mathrm{p}} / \mathrm{g}_{\mathrm{s}}$.

The Chern-Simons part of the action describes the interaction of the R-R fields as well as the interaction with the NS-NS fields

$$
S_{\mathrm{CS}}=\mu_{\mathrm{p}} \int_{D p} \sum_{q} \mathcal{P}\left[C_{(q+1)}\right] \wedge \operatorname{tr} \mathrm{e}^{\mathcal{F}} .
$$

The integral is restricted only to $p+1$-forms.

The DBI and CS part together form the relevant action for a single $D p$-brane at leading classical order in the string coupling $g_{s}$, that is at disk level, and at leading order in the derivatives.

Furthermore, the DBI and CS action can be generalized to coincident $D p$-branes, giving rise to the non-Abelian DBI and CS actions. Further details can be found in [29] or in the book [7]. Moreover, coincident $D p$-branes ending on Dq-branes $(q>p)$ can be described by excitations of massless fields on the higher-dimensional $D$-brane as well as by a dielectric effect on the lower-dimensional $D$-branes. For details involving coincident $D 1$-branes ending on a D3-brane see [30].

Besides BPS $D$-brane configurations there also exists non-BPS $D$-branes (for a review see [31]). A prominent example for non-BPS $D$-brane configurations are $D p-\overline{D p}$ systems, which are also important in brane-inflation models (see Chapter 2 for more details). Since $D p$ - and $\overline{D p}$-branes are oppositely charged, the branes attract each other. If the spatial distance between the $D p$ - and the anti- $D p$-brane is of the order of the string length, a tachyon [32] will be present in the spectrum of open strings. This tachyon indicates an instability of the $D p-\overline{D p}$-brane system; indeed the $D p$ - and anti $D p$-brane will annihilate to closed-string radiation.

The effective action of non-BPS D-brane configurations involves not only the massless string excitations but also the tachyon - a complex scalar field (for a proposal of an effective action for the $D p-\overline{D p}$ see [33]). 


\subsection{2}

\section{$D$-Branes as Charged BPS Objects}

In superstring theories $D p$-branes carry conserved charges of topological nature. In particular $D$-branes are described at low energies by solitonic supersymmetric solutions of the effective supergravity equations of motion that carry R-R charge, which is given by (1.149). Splitting the ten-dimensional spacetime into the worldvolume directions $a, b=0, \ldots, p$ and the transverse directions $i, j=p+1, \ldots, 9$ the solution for the metric, dilaton, and the $\mathrm{R}-\mathrm{R} p$-form potentials are given by

$$
\begin{aligned}
\mathrm{ds}^{2} & =H_{\mathrm{p}}^{-1 / 2} \eta_{a b} \mathrm{~d} x^{a} \mathrm{~d} x^{b}+H_{\mathrm{p}}^{1 / 2} \mathrm{~d} x^{i} \mathrm{~d} x^{i}, \\
\mathrm{e}^{\phi} & =g_{\mathrm{s}} H_{\mathrm{p}}^{(3-p) / 4}, \\
C_{(p+1)} & =\left(H_{\mathrm{p}}^{-1}-1\right) \mathrm{gs}_{\mathrm{s}}^{-1} \mathrm{~d} x^{0} \wedge \mathrm{d} x^{1} \wedge \cdots \wedge \mathrm{d} x^{p}, \\
B_{\mu \nu} & =0,
\end{aligned}
$$

where the harmonic function $H_{\mathrm{p}}(r)$ with $r^{2}=\delta_{i j} x^{i} x^{j}$ is

$$
H_{\mathrm{p}}=1+\left(\frac{r_{\mathrm{p}}}{r}\right)^{7-p}
$$

Whereas $C_{(q+1)}$ vanishes for a $D p$-brane $(q \neq p)$, the $D p$-brane induces a nontrivial $p+2$-form field strength $F_{(p+2)}=\mathrm{d} C_{(p+1)}$ as well as a nontrivial dilaton for $p \neq 3$. The charge of a $D p$-brane can be calculated by integrating the R-R flux through the $(8-p)$-dimensional sphere at infinity in the transversal dimensions,

$$
Q=\int_{S^{8-p}} \star F_{(p+2)},
$$

where $\star$ is the Hodge operator.

\section{9}

\section{Compactification}

We have seen that superstring theory is consistent in ten dimensions. Therefore, it remains necessary to find a way to obtain four-dimensional physics, and in particular the Standard Model of elementary particle physics, from superstring theory. For this purpose, two different approaches are pursued:

- Kaluza-Klein compactifications

In the Kaluza-Klein compactifications it is assumed that the ten-dimensional target spacetime of superstring theories has four uncompactified large dimensions, which correspond to the real world. The remaining six dimensions are compactified on a length scale $l_{\text {c }}$. For observations at energies $E \ll$ $1 / l_{c}$ the six compactified directions are invisible, though the topology and the length scales of the compactification have influence on the spectrum of the 
four-dimensional theory at low energies. We assume that the spacetime can be written as a product

$$
\mathcal{M}_{10}=\mathbb{R}^{3,1} \times \mathcal{M}_{6},
$$

where $\mathbb{R}^{3,1}$ is the four-dimensional Minkowski spacetime. Promising candidates for $\mathcal{M}_{6}$ are Calabi-Yau manifolds. Because of the special properties of these manifolds, the effective four-dimensional theory at low energies preserves some supersymmetry.

- Brane-world scenarios

In this approach the Minkowski spacetime of the four uncompactified dimensions is identified with a defect embedded into ten-dimensional spacetime. The defect is generated by intersecting and coincident $D$-branes. In brane-world models so-called warped compactifications are studied, where the length scale of the four-dimensional Minkowski spacetime depends on the coordinates of the compactified dimension. However, Poincaré invariance of the four-dimensional Minkowski spacetime is preserved. The typical metric of a warped compactification is given by

$$
\mathrm{d} s^{2}=\mathrm{e}^{2 A(\gamma)} g_{\mu \nu} \mathrm{d} x^{\mu} \mathrm{d} x^{\nu}+\mathrm{e}^{-2 A(\gamma)} \tilde{\mathrm{g}}_{a b} \mathrm{~d} y^{a} \mathrm{~d} \gamma^{b},
$$

where $g_{\mu \nu}$ is the metric of the four-dimensional spacetime. We see that in contrast to (1.150), the four and six-dimensional spaces are no longer topologically independent.

\subsection{1}

\section{String Theory on Calabi-Yau Manifolds}

Before we discuss features of string theory on Calabi-Yau manifolds, we have to define Kähler and Calabi-Yau manifolds. Kähler manifolds are complex manifolds for which the exterior derivative of the fundamental form, associated with the given Hermitean metric vanishes. The metric on the Kähler manifold locally satisfies

$$
G_{i \bar{j}}=\frac{\partial^{2} K}{\partial z^{i} \partial \bar{z}^{j}},
$$

where $K$ is the so-called Kähler function.

Calabi-Yau $n$-folds are Kähler manifolds in $n$ complex dimensions with the additional topological property of vanishing first Chern class, $c_{1}=0$. Any compact Kähler manifold with $c_{1}=0$ admits a Kähler metric with $S U(n)$ holonomy and is necessarily Ricci flat. See [34] for the precise definition. Examples of Calabi-Yau manifolds are

- The complex plane $\mathbb{C}$ and the two-torus $T^{2}$ in two (real) dimensions. These are all examples in two dimensions since any compact Riemannian surface, except the torus $T^{2}$, is not a Calabi-Yau manifold.

- In four (real) dimensions there are two compact Calabi-Yau manifolds: the four-torus $\mathrm{T}^{4}$ and K3. Examples of noncompact Calabi-Yau manifolds are $\mathbb{C}^{2} \times T^{2}$ and $\mathbb{C}^{4}$. 
- In six (real) dimensions there are many Calabi-Yau three-folds known. In fact, the number of Calabi-Yau three-folds may even be infinite since there is no mathematical proof for the finiteness of the number up to now.

\subsubsection{1}

\section{Low-Energy Effective Theory}

Let us discuss the properties of four-dimensional gauge theories which are obtained from compactifications. Compactifying ten-dimensional superstring theory on Calabi-Yau three-folds breaks 3/4 of supersymmetry. Therefore the effective four-dimensional theory has $\mathcal{N}=1$ supersymmetry for heterotic string theories and $\mathcal{N}=2$ for type II superstring theories. The low-energy effective theory in four spacetime dimensions for type II superstring theories consists of

$-\mathcal{N}=2$ supergravity multiplet,

$-\mathcal{N}=2$ Abelian vector multiplets,

$-\mathcal{N}=2$ hypermultiplets.

The number of vector and hypermultiplets depend on topological properties of the Calabi-Yau manifolds, in particular on the Hodge numbers $h^{p, q}$.

By adding $D$-branes to the compactification setup we may obtain non-Abelian gauge theories in four dimensions. Compactifications of type II superstring theories are often inconsistent or unstable due to uncanceled charges of $D$-branes or due to fluxes. A remedy is to perform a orientifold projection ${ }^{10)}$. In particular the charges and tensions of $D$-branes are canceled by orientifold-planes (so-called $O$ planes). Orientifold projections ensure that the four-dimensional low-energy effective action preserves $\mathcal{N}=1$ supersymmetry. The bosonic part of $\mathcal{N}=1$ supergravity coupled to chiral and vector multiplets is given by

$$
\begin{aligned}
\mathcal{L}=\frac{1}{2 \kappa_{4}} R & -G_{i \bar{j}}(\phi, \bar{\phi}) D_{\mu} \phi^{i} D^{\mu} \bar{\phi}^{\bar{j}}-\mathcal{V} \\
& -\frac{1}{8} \operatorname{Re} f_{a b}(\phi) F_{\mu \nu}^{a} F^{b \mu \nu}-\frac{1}{8} \operatorname{Im} f_{a b}(\phi) \varepsilon^{\mu \nu \varrho \sigma} F_{\mu \nu}^{a} F_{\varrho \sigma}^{b} .
\end{aligned}
$$

$\phi^{i}$ are the scalar fields of the chiral multiplet and $F_{\mu \nu}$ is the field strength tensor of the gauge fields of the vector multiplet. Moreover, $D_{\mu}$ is the covariant derivative and $G_{i j}$ is the metric of the scalar target space ${ }^{11)}$, which is given by the Kähler potential $K(\phi, \bar{\phi})$,

$$
G_{i \bar{j}}=\frac{\partial^{2} K(\phi, \bar{\phi})}{\partial \phi^{i} \partial \bar{\phi}^{\bar{j}}}
$$

The gauge-kinetic function $f_{a b}(\phi)$ in (1.153) is an arbitrary analytic function of the scalar fields $\phi^{i}$. If some scalar fields $\phi^{i}$ acquire vacuum expectation values dynamically, this may generate vacuum expectation values for $f_{a b}(\phi)$. This controls the

10) For more details see [35].

11) The inverse matrix of $G_{i j}$ is as usual denoted by upper indices. 
coupling constant $g_{a b}$ of the gauge fields ${ }^{12)}$, since

$$
\operatorname{Re} f_{a b}=g_{a b}^{-2} \text {. }
$$

The last ingredient of the general form of $\mathcal{N}=1$ supergravity in four spacetime dimensions is the scalar potential $\mathcal{V}$, which consists of two parts referred to as Dand F-term potentials:

$$
\mathcal{V}=\mathcal{V}_{\mathrm{F}}+\mathcal{V}_{\mathrm{D}}
$$

The F-term potential is determined by the holomorphic superpotential $\mathcal{W}(\phi)$,

$$
\mathcal{V}_{\mathrm{F}}=\mathrm{e}^{K / M_{\mathrm{pl}}^{2}}\left(G^{i \bar{j}} D_{i} \mathcal{W} D_{\mathrm{j}} \overline{\mathcal{W}}-3 M_{\mathrm{pl}}^{2}|\mathcal{W}|^{2}\right)
$$

where the covariant derivative of the superpotential is defined by

$$
D_{i} \mathcal{W}=\partial_{i} \mathcal{W}+M_{\mathrm{pl}}^{2} G_{i} \mathcal{W} \quad \text { with } \quad G_{i}=\frac{\partial K(\phi, \bar{\phi})}{\partial \phi^{i}}
$$

The D-term potential $\mathcal{V}_{\mathrm{D}}$ is expressed in terms of the auxiliary $D^{a}$-fields of the vector multiplet,

$$
\mathcal{V}_{\mathrm{D}}=\frac{1}{2}\left(\operatorname{Re}(f)^{-1}\right)_{a b} D^{a} D^{b}
$$

In contrast to global supersymmetric field theories, the scalar potential $\mathcal{V}$ does not have to be zero in a supersymmetry preserving vacuum since a supersymmetric vacuum has to satisfy $D^{a}=D_{i} \mathcal{W}=0$. Therefore, the scalar potential (1.157), (1.158) in a supersymmetric vacuum has the value

$$
\mathcal{V}=-3 M_{\mathrm{pl}}^{2}|\mathcal{W}|^{2} \exp \left(K / M_{\mathrm{pl}}^{2}\right)
$$

Since the scalar potential is negative and the scalar potential is related to the cosmological constant, the vacuum has negative cosmological constant. This is good news, since states with broken supersymmetry (i.e. $D^{a} \neq 0$ or $D_{i} \mathcal{W} \neq 0$ ) can have an almost zero scalar potential and therefore a tiny positive cosmological constant.

\section{9 .2}

\section{String Theory on Orbifolds}

Even for simple examples of Calabi-Yau manifolds (for example K3), the metric is not known explicitly. Therefore, one generally considers string theory on orbifolds since there effects of singular limits of Calabi-Yau manifolds can be studied. Moreover, the metric of orbifolds is explicitly known. For more details see the review [35].

The definition of an orbifold is as follows. Let $X$ be a smooth manifold with a discrete isometry group $G$. An orbifold is the quotient space $X / G$. The points of $X / G$ are precisely the points of $X$, whereas two points $x_{1}$ and $x_{2}$ are identified if they are connected by a group transformation. Simple examples are

12) In renormalizable field theories we have only one gauge coupling for the gauge fields since $\mathrm{g}_{a b}$, i.e. $\mathrm{g}_{a b}=\mathrm{g} \delta_{a b}$. 
- $S^{1} / \mathbb{Z}_{2}$, that is opposite points on a circle will be identified. This gives an interval.

- The orbifold $\mathbb{C} / \mathbb{Z}_{N}$ is a cone, since points of the complex plane which can be mapped onto each other by a rotation by $(2 \pi k) / N$ are identified.

We see that orbifolds can be singular. This generally happens if nontrivial group elements leave points of $X$ invariant. Although general relativity is ill-defined on singular spaces, it is possible to define string theory consistently on orbifolds. There are two types of strings on orbifolds:

- untwisted states, that is states which exist on $X$ and are invariant under the group G.

- twisted states are new closed-string states, which satisfy the condition $X^{\mu}(\tau, \sigma+$ $2 \pi)=g X^{\mu}(\tau, \sigma)$, where $g \in G$. The twisted sectors are labeled by the particular group element $g$. Untwisted strings correspond to $g=1$. A generic feature of orbifolds is the localization of strings of the twisted sector at the orbifold singularities.

Only by including twisted states into the string spectrum can strings propagate consistently on spaces with orbifold singularities. Furthermore, string theories on orbifolds $X / G$ have more broken supersymmetry than on $X$, which again makes them interesting for the phenomenology of string theory.

\section{9 .3}

\section{String Moduli and Their Stabilization}

Some of the Calabi-Yau manifolds are smoothly related by deformations of parameters characterizing the shape and size. These parameters are called string moduli. Examples are expectation values of the dilaton field, the values of coupling constants, expectation values of Wilson lines around nontrivial cycles and various parameters (e.g. the radii and the complex structure) describing the shape of the compactification manifold.

As a simple example let us discuss the moduli space of bosonic string theory compactified on a circle $S^{1}$ along the $X^{25}$-direction. In that case the relevant parameter describing the compactification is the radius $R$ of the compactified direction. Whereas in field theory every radius leads to different physics, the situation in string theory is different due to T-duality (see Section 1.7.2). Backgrounds with large radius (in string units) are transformed into backgrounds with small radius $\tilde{R}$ (in string units). Therefore, there exists a smallest (respectively biggest) radius $R_{0}=\sqrt{\alpha^{\prime}}$ and the moduli space is $\mathcal{M}=\left\{R \leq R_{0}\right\}$ or equivalently $\mathcal{M}=\left\{R \geq R_{0}\right\}$. In a more formal way the moduli space can be written in the form

$$
\mathcal{M}=\left\{R \in \mathbb{R}_{+} / \mathbb{Z}_{2}\right\}
$$

In string theory the parameters of the moduli space describe different vacuum configurations and can be related to vacuum expectation values of massless scalar fields. In the case of a circle compactification the vacuum expectation value of the 
scalar

$$
|\Phi\rangle=\alpha_{-1}^{25} \tilde{\alpha}_{-1}^{25}|0\rangle
$$

is related to the radius of the circle.

This is a general rule of perturbative string theory: moduli can be considered as massless scalar fields on the string world-sheet. However, it is not possible to predict the vacuum expectation value of the moduli, since the potential is flat in the four-dimensional effective theory. This is the moduli stabilization problem. One possibility to solve it is to use warped background compactifications. Nontrivial warp factors in the metric are obtained by nonvanishing fluxes, by branes, and by higher-order corrections to low-energy supergravity actions. For details see Chapters 2 and 3 in this book or the review [35].

\subsection{0}

\section{String Thermodynamics}

In this section, we introduce thermodynamics of open bosonic strings ending on a $D p$-brane. For simplicity we consider only bosonic open strings that do not carry spatial momentum. To compute the entropy in the microcanonical ensemble, we have to count the string states with given energy $E$. This is done by determining all partitions of the string level number $N$ associated with the string energy $E=$ $\sqrt{N-1} / \sqrt{\alpha^{\prime}}$. The result for the number of string states is (see also [2])

$$
\Omega(E) \simeq E^{-\gamma} \exp \left(4 \pi \sqrt{\alpha^{\prime}} E\right),
$$

with $\gamma=(25-p) / 2$. Calculating the entropy by using $S(E)=k_{\mathrm{B}} \ln \Omega(E)$, we obtain a relation between the entropy $S$ and the energy $E$ which is asymptotically linear for high energies $E \gg 1$,

$$
S(E)=-\gamma k_{\mathrm{B}} \ln E+4 \pi \sqrt{\alpha^{\prime}} k_{\mathrm{B}} E .
$$

This is an unusual behavior in statistical mechanics since it immediately leads to a constant temperature at high energies,

$$
\frac{1}{\beta}=k_{\mathrm{B}}^{-1} \frac{\partial S}{\partial E}=-\frac{\gamma}{E}+4 \pi \sqrt{\alpha^{\prime}}
$$

which is called the Hagedorn temperature $T_{\mathrm{H}}$ and is given by $T_{\mathrm{H}}=\left(4 \pi \sqrt{\alpha^{\prime}} k_{\mathrm{B}}\right)^{-1}$. Inverting the equation (1.165) we obtain

$$
E(T)=\gamma\left(\frac{1}{k_{\mathrm{B}} T_{\mathrm{H}}}-\frac{1}{k_{\mathrm{B}} T}\right)^{-1} .
$$

For $T \rightarrow T_{\mathrm{H}}$ the energy $E$ diverges. For closed strings and open superstrings the same relation up to numerical factors hold. 
In the canonical ensemble the string partition function $Z_{\text {string }}$ also diverges if the temperature $T$ approaches the Hagedorn temperature. The partition function is well approximated by

$$
Z_{\text {string }} \simeq \frac{C}{T_{H}-T}
$$

for $T \rightarrow T_{\mathrm{H}}$. For more details and computations see for example [2].

\subsection{1}

\section{Gauge-Gravity Duality}

Gauge-gravity dualities are dualities between a (quantum) theory of gravity on one hand, and a gauge theory in a spacetime with a lower number of dimensions on the other. Gauge-gravity dualities may be viewed as a realization of the holographic principle, since the same amount of degrees of freedom is encoded in the higherdimensional space as well as in the lower-dimensional. There is a one-to-one correspondence between gauge invariant operators in the gauge theory and fields in the dual gravity theory. Moreover, gauge-gravity dualities are strong-weak coupling dualities in the sense that one theory is strongly coupled, whereas the other theory is weakly coupled and therefore accessible to perturbation theory. For instance, by applying gauge-gravity dualities, strongly coupled gauge theories can be investigated by mapping them to weakly coupled gravity theories. The most prominent example of gauge-gravity duality is the duality between type IIB supergravity on a five-dimensional Anti-de Sitter (AdS) spacetime and $\mathcal{N}=4 S U(N)$ super YangMills theory on the conformal boundary of the Anti-de Sitter spacetime. This is the well-known AdS/CFT correspondence conjectured by Maldacena in 1997 [36], in which the conformal field theory is $\mathcal{N}=4 S U(N)$ super Yang-Mills.

The duality is motivated by looking at the two different aspects of $D$-branes as described in Section 1.8. On one hand $D$-branes can be viewed as hyperplanes where open strings end. The low-energy effective dynamics of open strings is described by a gauge theory. On the other hand D-branes are charged BPS solutions of supergravity curving the surrounding spacetime. For the duality involving $\mathcal{N}=4$ Yang-Mills theory described above, the map between gauge and gravity theory is obtained by considering the two aspects for the case of D3-branes: in a particular low-energy decoupling limit [36], open strings ending on D3-branes give rise to $\mathcal{N}=4$ super Yang-Mills theory on four-dimensional Minkowski spacetime. In the supergravity picture, the low-energy limit gives rise to closed-string excitations in the space $A d S_{5} \times S^{5}$. The degrees of freedom in both pictures are then identified.

This leads to the conjecture that gravitational theories and gauge theories describe the same physics from different points-of-view. The interested reader is referred to the reviews $[37,38]$ for further details. In Chapter 7 , gauge-gravity dualities are applied to string cosmology. 


\section{Summary}

We have presented essential features of both cosmology and string theory which will be used in the subsequent chapters where string cosmology is introduced. With the material presented in this chapter, the reader is now equipped for studying the string cosmology research topics in the subsequent chapters.

\section{References}

1 V. Mukhanov, Physical Foundations of Cosmology. Cambridge University Press, 2005.

2 B. Zwiebach, A first course in string theory, Cambridge University Press (2004), $558 \mathrm{p}$.

3 E. Kiritsis, String theory in a nutshell, Princeton University Press (2007), 588 p.

4 K. Becker, M. Becker, and J.H. Schwarz, String theory and M-theory: A modern introduction, Cambridge University Press (2007), $739 \mathrm{p}$.

5 M.B. Green, J.H. Schwarz, and E. Witten, Superstring theory, Vol. I and Vol. II. Cambridge University Press, 1987.

6 J. Polchinski, String theory, Vol. I and Vol. II. Cambridge University Press (1998), $402 \mathrm{p}$.

7 C.V. Johnson, D-branes. Cambridge University Press, 2003.

8 L. McAllister and E. Silverstein, String Cosmology: A Review, Gen. Rel. Grav. 40 (2008) 565 [arXiv:0710.2951 [hep-th]].

9 C.P. Burgess, Lectures on Cosmic Inflation and its Potential Stringy Realizations, PoS P2GC (2006) 008 [Class. Quant. Grav. 24 (2007) S795] [arXiv:0708.2865 [hep-th]].

10 J.M. Cline, String cosmology, Lectures given at Advanced Summer Institute on New Trends in Particle Physics and Cosmology, Sheffield, England, 19-23 June 2006 and at Les Houches Summer School - Session 86: Particle Physics and Cosmology: The Fabric of Spacetime, Les Houches, France, 31 Jul-25 Aug 2006. arXiv:hep-th/0612129 (www.arXiv.org).
11 M. Trodden and S.M. Carroll, TASI lectures: Introduction to cosmology, arXiv:astro-ph/0401547 (www.arXiv.org).

12 M. Gasperini: Elements of String Cosmology. Cambridge University Press, 2007.

13 A.A. Penzias and R.W. Wilson, Astrophys. J. 142 (1965) 419.

14 E. Komatsu et al. [WMAP Collaboration], "Five-Year Wilkinson Microwave Anisotropy Probe (WMAP) Observations: Cosmological Interpretation", arXiv:0803.0547 [astro-ph] (www.arXiv.org); K. A. Olive, TASI lectures: Dark matter, arXiv:astro-ph/0301505 (www.arXiv.org).

15 A.H. Guth, Phys. Rev. D 23, 347 (1981); A.D. Linde, Phys. Lett. B 108, 389 (1982); A. Albrecht and P.J. Steinhardt, Phys. Rev. Lett. 48, 1220 (1982).

16 P.J.E. Peebles: The Large-Scale Structure of the Universe, Princeton University Press, 1980.

17 A. Sen and B. Zwiebach, Tachyon condensation in string field theory, JHEP 0003 (2000) 002.

18 E. D'Hoker and D.H. Phong, The Geometry of String Perturbation Theory, Rev. Mod. Phys. 60 (1988) 917.

19 R.G. Leigh, Dirac-Born-Infeld Action from Dirichlet Sigma Model, Mod. Phys. Lett. A 4 (1989) 2767.

20 N. Berkovits, ICTP lectures on covariant quantization of the superstring, arXiv:hepth/0209059.

21 F. Gliozzi, J. Scherk, and D.I. Olive, Supersymmetry, Supergravity Theories And The Dual Spinor Model, Nucl. Phys. B 122 (1977) 253. 
22 J. Dai, R.G. Leigh and J. Polchinski, New Connections Between String Theories, Mod. Phys. Lett. A 4 (1989) 2073.

23 C.M. Hull and P.K. Townsend, Unity of superstring dualities, Nucl. Phys. B 438, 109 (1995).

24 E. Witten, String theory dynamics in various dimensions, Nucl. Phys. B 443 (1995) 85 [arXiv:hep-th/9503124].

25 N.A. Obers and B. Pioline, U-duality and M-theory, Phys. Rept. 318, 113 (1999)

26 P.K. Townsend, The eleven-dimensional supermembrane revisited, Phys. Lett. B 350 (1995) 184 [arXiv:hep-th/9501068].

27 P. Horava and E. Witten, Heterotic and type I string dynamics from eleven dimensions, Nucl. Phys. B 460 (1996) 506 [arXiv:hep-th/9510209].

28 J. Polchinski, Dirichlet-Branes and Ramond-Ramond Charges, Phys. Rev. Lett. 75 (1995) 4724 [arXiv:hepth/9510017].

29 R.C. Myers, Dielectric-branes, JHEP 9912 (1999) 022 [arXiv:hep-th/9910053]

30 N.R. Constable, R.C. Myers and O. Tafjord, The noncommutative bion core, Phys. Rev. D 61 (2000) 106009 [arXiv:hep-th/9911136].
31 J.H. Schwarz, TASI lectures on non-BPS D-brane systems, arXiv:hep-th/9908144.

32 T. Banks and L. Susskind, Brane Antibrane Forces, arXiv:hep-th/9511194.

33 P. Kraus and F. Larsen, Boundary string field theory of the DD-bar system, Phys. Rev. D 63 (2001) 106004 [arXiv:hepth/0012198]

34 B.R. Greene, String theory on Calabi-Yau manifolds, arXiv:hep-th/9702155.

35 R. Blumenhagen, B. Körs, D. Lüst and S. Stieberger, Four-dimensional String Compactifications with D-Branes, Orientifolds and Fluxes, Phys. Rept. 445, 1 (2007)

36 J.M. Maldacena, The large $N$ limit of superconformal field theories and supergravity, Adv. Theor. Math. Phys. 2 (1998) 231

37 O. Aharony, S.S. Gubser, J.M. Maldacena, H. Ooguri and Y. Oz, Large N field theories, string theory and gravity, Phys. Rept. 323 (2000) 183 [arXiv:hepth/9905111].

38 E. D'Hoker and D.Z. Freedman, Supersymmetric gauge theories and the AdS/CFT correspondence, arXiv:hep-th/0201253. 